\title{
Die Welt der Kunst: Formen der Spezifikation des Beobachtens
}

\author{
https://doi.org/10.1515/sosys-2019-0014
}

Zusammenfassung: Eine angemessene theoretische Beschreibung der Kunst muss sich von den philosophischen Traditionen lösen. Dies geschieht durch einen Ansatz, der von dem unterscheidungstheoretischen Begriff der Beobachtung und darauf aufbauend dem Konzept der Beobachtung 2. Ordnung ausgeht. Auf diese Weise können neue Ansätze einer Theorie der Ästhetik skizziert werden, für die ein entsprechender Begriff der Wahrnehmung zentral ist. Wahrnehmung kann als eine besonders kompakte, unterscheidungsbezogen simultane Form der Beobachtung beschrieben werden. Die Wahrnehmung eines Kunstwerks ist eine dritte Möglichkeit des Beobachtens: das Kunstwerk benutzt eine Unterscheidung, um die eine und nicht die andere Seite zu fokussieren, diese aber gleichzeitig mitzuzeigen, und es benutzt eine Vielzahl von aufeinander verweisenden Unterscheidungen gleichzeitig. Dies führt zu einer Erstaunlichkeit des Kunstwerks und stellt besondere Anforderungen an den Beobachter von Kunst. Diesen Beobachter der Kunst und die von ihm vorausgesetzten Unterscheidungen kann wiederum ein Beobachter - die Literatur, die Psychologie, die Soziologie - beobachten. Die Erstaunlichkeit des Kunstwerks ist Anlass für und ist ein Medium der Kommunikation über Kunst, da verschiedene Beobachter Verschiedenes sehen. Entsprechend kann man Zusammenhänge zwischen dem Formangebot der Kunstwerke und der Ausdifferenzierung der Kunst als soziales System identifizieren. Abschließend wird auf dieser Basis nach der binären Codierung des gesellschaftlichen Funktionssystems Kunst gefragt.

Abstract: An adequate theoretical description of art must break away from philosophical traditions. This is achieved through an approach that starts from the distinction-theoretical concept of observation and the concept of second order observation. In this way, new approaches to a theory of aesthetics can be outlined, for which a corresponding concept of perception is central. Perception can be described as a particularly compact, distinction-related simultaneous form of observation. The perception of a artwork is a third way of observing: the work of art uses a distinction to focus on one side of the distinction and not the other, but at the same time it also shows it, and it uses a multiplicity of distinctions referring to each other at the same time. This leads to an astonishment of the artwork and makes special demands on the observer of art. This observer of art and the distinctions it presupposes can in turn be observed by an observer - literature, psychology, sociology. The amazingness of the artwork is the occasion for and 
is a medium of communication about art, since different observers see different things. Accordingly, one can identify connections between the form offered by artworks and the differentiation of art as a social system. Finally, on this basis, we ask about the binary code of the social functional system of art.

\section{I.}

Theoretische Beschreibungen der Kunst sind durch die philosophische Tradition nicht gerade begünstigt worden. Die Philosophie hatte ihr eigenes Werk zu vollbringen. Sie hatte die Welt zu beschreiben, und zwar, nach traditioneller Vorstellung, im Hinblick auf ihr Sein im Unterschied zu Nichtsein. Dabei ging es, wie bei jeder sophía, zunächst einfach um die Herstellung eines guten Werkes, einer zutreffenden Beschreibung. Die Aufgabe war, eine richtige (und nicht eine falsche) Beschreibung zu liefern. Das führte auf die Frage der Kriterien und so auf den Abweg der Skepsis und in die Reflexion, mit der das Unternehmen der Beobachtung und Beschreibung der Welt begann, sich selbst zu beobachten und zu beschreiben. Auch dafür blieb die Frage, ob zutreffend oder unzutreffend, ob wahr oder unwahr, die Leitdifferenz. Und das Problem war noch bei Descartes, ob nicht alles Illusion, Phantasie, Traum, Einbildung sei und wie man die Weltkenntnis davon unterscheiden könne.

In dieser Tradition hatte das, was wir heute Kunst nennen, einen schlechten Stand. Kunst wurde im einfachsten Falle im Schema der Philosophie als Täuschung verstanden. Wo dieses Urteil zu hart erschien, konnte man der Täuschung einen positiven, zum Beispiel pädagogischen Sinn zuerkennen. Man mochte ihr eine Enklave zuweisen, in der unter Suspension der Wahrheitsfrage gespielt werden durfte. Bis ins 18. Jahrhundert hinein findet man entsprechende Diskussionen über das Verhältnis von Philosophie und Poesie. ${ }^{1}$ Zugleich erschien als eine Art Seitentrieb der Philosophie das, was man in einem neuen Sinne „Ästhetik“ nannte. In diesen Bereich strömten all die Impulse, die im traditionellen Wahrheitsschematismus der Philosophie und in dessen Reflexion nicht zur Geltung gekommen waren. Die Abhängigkeit von der Philosophie blieb gleichwohl erhalten und zeigte sich nun an den Übertreibungen einer Gegenbegrifflichkeit. Ein neuer ästhetischer Staat, eine neue Mythologie, ein neuer Mensch, neue Werte, neue Verzweiflungen wurden der Kunst zugemutet, so als ob sie sich in einem kompensatorischen Verhältnis zu den dominanten Formen des Vorstellens und Herstellens von Welt entfalten müsste. Und wenn auch dies noch reflektiert

1 Vgl. etwa d'Alembert 1967. 
wird, etwa bei Heidegger, dann wiederum in Abhängigkeit (wenngleich: in Kritik der Abhängigkeit) von der Prägung der Philosophie durch die ontologische Metaphysik, die an ihr Ende gekommen zu sein scheint. ${ }^{2}$

Dass in dieser Tradition bewahrenswerte Erkenntnisgewinne stecken, darf man vermuten. Um sie herauszubringen, bedarf es aber eines andersartigen Ansatzes. Wir benötigen eine Position, von der aus wir den Dialog zwischen Poesie und Philosophie beobachten können, und diese Position kann keine philosophische sein. Wenn die Aufgabe so gestellt ist, geht es um ein Beobachten von Beobachtungen, um ein Beobachten zweiter Ordnung oder eventuell um eine Kybernetik zweiter Ordnung. ${ }^{3}$ Wir müssen beobachten und beschreiben können, wie wir beobachten, wenn wir beobachten, und was wir beobachten, wenn wir beobachten, dass andere beobachten. Das macht es erforderlich, den Begriff der Beobachtung entsprechend zu abstrahieren.

\section{II.}

Unter Beobachtung soll hier eine Operation verstanden werden, die eine Unterscheidung spezifiziert, um die eine und nicht die andere Seite $\mathrm{zu}$ bezeichnen. Gegenüber der traditionellen Vorherrschaft erkenntnistheoretischer Fragen muss vor allem klargestellt werden, dass es sich hierbei nicht notwendigerweise um die Unterscheidung wahr/falsch handeln muss; es muss sich nur um irgendeine Unterscheidung handeln. Der Begriff ist also sehr viel abstrakter gefasst als der Begriff der Erkenntnis. Ein an Erkenntnis interessiertes Beobachten ist ein Sonderfall - und wie man wohl vermuten darf: ein relativ seltener Sonderfall.

Unterscheiden und Bezeichnen müssen dabei als zwei Komponenten einer einzigen Operation aufgefasst werden; sie lassen sich isoliert denken, auch isoliert vollziehen, aber das ist dann jeweils eine Abstraktion, die sich selbst daran hindert, sich als Beobachtung zu vollenden. Jede vollständige Beobachtung setzt etwas voraus, wovon sie sich abhebt. Sie unterscheidet, wenn man so sagen darf, indem sie etwas meint, intendiert, bezeichnet. Man kann einerseits sagen, dass jede Bezeichnung eine sie führende Unterscheidung voraussetzt. Andererseits gilt auch, dass jede Bezeichnung eine Selektion ist, die durch die Gegenseite des Nichtbezeichneten austariert werden muss, also eine Unterscheidung produziert. In diesem Sinne sind Unterscheidungen selbstkompensatorische Verhältnisse: sie gleichen eine Abstraktion durch eine Gegenabstraktion aus. In Wirklichkeit

2 Siehe nur Heidegger 1950.

3 Siehe hierzu Maturana 1982, insb. 36 f.; von Foerster 1981. 
liegt daher ein doppelt-zirkuläres Verhältnis vor: Die Bezeichnung erfordert die Unterscheidung und umgekehrt, weil die Bezeichnung die Nichtbezeichnung erfordert und umgekehrt. Das berechtigt uns, Unterscheiden und Bezeichnen mit George Spencer Brown (1979) als eine einheitliche Operation anzusehen; und eben diese Operation nennen wir Beobachten.

Jede Unterscheidung ist, auch und gerade wenn sie einen Bereich von Möglichkeiten komplett definiert, eine Reduktion von Komplexität. ${ }^{4}$ Es gibt keine Unterscheidung, für die dies nicht gälte. Eben deshalb ist die Welt selbst nicht Gegenstand einer möglichen Unterscheidung, deshalb auch nichts, was man bezeichnen könnte. ${ }^{5}$ Daraus ergibt sich zugleich, dass jede Beobachtung einen Strukturgewinn aktualisiert (mag er nun „neu“ sein oder nicht). Jede Beobachtung ist, da sie eine Unterscheidung mitreproduziert, strukturgebende Selektion, und nur als Moment der Unterscheidung, die sie verwendet, ist sie wiederholbar. Im Prozessieren von Beobachtungen werden die sie ermöglichenden Unterscheidungen laufend gewechselt. Strukturen sind also zunächst nahezu so labil wie die Ereignisse selbst, und beides zusammen wechselt im Prozessieren des Erlebens und Handelns. Dennoch ist die Unterscheidung von Bezeichnung und Unterscheidung, von Ereignis und Struktur von fundamentaler Bedeutung; denn Ereignisse vergehen zwangsläufig, sie verschwinden, sobald sie aktualisiert werden; Strukturen haben dagegen zumindest die Chance der Stabilität. Unterscheidungen ermöglichen, indem sie identisch gehalten werden, das Wiederholen, Bestätigen, Kondensieren des beobachteten Sinnes; sie ermöglichen die Rückkehr zum Selben, und sie ermöglichen den Übergang zum Gegenteil - etwa die Einsicht, dass das, was nützlich zu sein schien, schädlich ist.

Mit anderen Worten kann man auch sagen: Aller Sinn wird binär prozessiert, wird von einem unverlierbaren Schatten des Negativen begleitet, mit der stets mitlaufenden Möglichkeit, das Negative durch Negation seiner Negativität zu positivieren oder es auch nur zu benutzen, um das Positive von da aus zu sehen. In diesem Sinne ist es Schicksal der Frau, kein Mann zu sein. Dies Schicksal lässt sich vermeiden - aber nur, wenn man darauf verzichtet, die Unterscheidung von Mann und Frau zur Bezeichnung der Frau zu verwenden. Dies ist jedoch

4 Frühe und stimulierende Bemerkungen zu dieser heute geläufigen Einsicht finden sich im Kapitel „Scope and Reduction“ bei Burke 1962, 59ff., insb. 96: „To note any order whatsoever is to ,reduce'. To divide experience into hungry and sated moments, into the pleasant and unpleasant, into the before and after, into here and there - even distinctions so broad as these translate the world's infinite particulars into terms that are a reduction of the world; in fact, as per the equating of infinity and zero, terms of such broad scope are perhaps the most drastically reductive of all“.

5 Das schließt selbstverständlich Kosmologien nicht aus; denn jede Kosmologie ist selbst eine Reduktion, ,a reduction of the world to the dimensions of words“ (Burke 1962, 96). 
nur möglich, wenn man eine andere Unterscheidung substituiert - etwa die von Frau und Weiblichkeit; ${ }^{6}$ oder wenn man sich (oder ihr) es zutraut, die binäre Unterscheidung mit einem Wert $\mathrm{zu}$ versehen, der weder das Bezeichnete noch das Nichtbezeichnete, sondern die Selbstbezeichnung markiert. ${ }^{7}$

Aus diesem Begriff des Beobachtens lässt sich selbstverständlich nicht folgern, was beobachtet wird; geschweige denn: was beobachtet werden sollte. Die Leistung des Begriffs besteht nur darin, die Aufmerksamkeit auf eine Vorfrage $\mathrm{zu}$ lenken, deren Beantwortung vielleicht wichtiger ist, nämlich auf die Frage: mit Hilfe welcher Unterscheidung beobachtet wird; oder schwieriger noch: wie es um die Unterscheidung von Unterscheidungen oder wie es um die Rationalität der Wahl einer Unterscheidung steht. ${ }^{8}$ Man kann zum Beispiel fragen, was geschieht, wenn eine Gesellschaft ihre Selbstbeschreibung von Natur/Gnade auf Natur/Zivilisation umstellt oder von honestas/utilitas auf nützlich/nutzlos bzw. nützlich/schädlich - beides offensichtlich semantische Veränderungen des 18. Jahrhunderts im Zuge der Selbstverständigung über moderne Gesellschaft.

Eine Unterscheidung, die beim Beobachten zur Bezeichnung von etwas verwendet wird, soll Kontext der Beobachtung genannt werden, um deutlich zu machen, dass nichtintendierte Bedingungen mitspielen. Kontext heißt dann immer auch: dass dritte Möglichkeiten ausgeschlossen sind. ${ }^{9}$ Jede Unterscheidung impliziert damit die Möglichkeit anderer Unterscheidungen, indem sie es

6 Vgl. dazu Meyer 1983.

7 Überlegungen in dieser Richtung bei Varela 1975.

8 Formal ist dieses Problem durchaus bekannt. Gotthard Günther z. B. spricht von einer „tieferen Zweiwertigkeit“, von der Unterscheidung von Akzeptions- und Rejektionswert in Bezug auf die Wahl eines zweiwertigen Schematismus (siehe Günter 1976a, 231). Ein anderer Weg ist: die erste Unterscheidung mit der in sie eingebauten Unterscheidung von Unterscheidung und Bezeichnung als zwangsläufig-willkürlich zu setzen und aus den dadurch mitgesetzten Implikationen einen rationalen Kalkül zu entwickeln. Dass das geht, hat George Spencer Brown (1979) gezeigt. 9 Entsprechend definiert Gotthard Günther $(1978,290)$ Kontextur durch die Operation des Ausschlusses von dritten Möglichkeiten. Damit inkonsistent ist allerdings die Gewohnheit Günthers, Sein und Nichtsein als verschiedene Kontexturen anzusehen. Vor allem aber müssen wir in einer anderen Hinsicht abweichen: Bei Günther wird der Begriff der Kontextur strikt auf der logischen Ebene gehalten, was einen (gänzlich unrealistischen) Begriff des psychischen Systems erfordert, der dieses auf ein geschlossen-zweiwertiges Operieren beschränkt mit der Folge, dass Mehrwertigkeit nur für eine Mehrheit von Subjekten zum Problem wird. Um diese (für Wahrnehmungsprozesse fatale) Einschränkung zu vermeiden, generalisieren wir den Begriff des Kontextes unter Einschluss dessen, was Günther „partial negations“ nennt (287) und beziehen ihn auf jede Art von Unterscheidung, die zur Beobachtung verwendet wird, sofern nur diese Unterscheidung Drittes ausschließt und dadurch von anderen Unterscheidungen unterscheidbar ist. Um Verwechselungen zu vermeiden, bleiben wir daher beim Begriff des Kontextes (statt Günthers „Kontextur“). 
zugleich ausschließt, dass sie im Kontext der eigenen Operationen verwendet werden.

Geht man in dieser Weise vom Unterscheiden aus, weil das Ausgehen selbst schon ein Unterscheiden ist, verändert das den Begriff der Welt. Er kann, und muss, für das Nichtunterscheidbare reserviert werden. Die Welt, das ist das, wovon man sich nicht unterscheiden kann. Alles Unterscheiden reorganisiert die Welt, indem man sie wiederfindet als das, was die Unterscheidung als Einheit der Differenz voraussetzt. In jeder Unterscheidung ist die Welt das eingeschlossene ausgeschlossene Dritte. Auch im Unterscheiden von Unterscheidungen wiederholt sich diese Reformierung der Welt, gesehen als Einheit jeweils anderer Unterscheidungen. Das heißt auch, dass man zur Welt keine Beziehungen herstellen kann, denn auch das würde voraussetzen, dass man die Welt von etwas anderem unterscheiden kann. Ferner besagt dieser Weltbegriff, dass die Welt nicht bezeichnet werden kann, denn das würde eine Unterscheidung voraussetzen, in der die Welt das eine und nicht das andere ist. Die Welt kann nur symbolisiert bzw. diabolisiert werden je nachdem, ob man an der Unterscheidung, von der aus die Welt gesehen wird, zum Beispiel an der Unterscheidung von gut und böse, das Zusammen oder das Auseinander betont, wobei die Welt auch dann wiederum die Einheit dieser Differenz ist. Es gibt daher auch keine Mehrheit von Welten, sondern nur eine polykontexturelle Welt, ${ }^{10}$ die freilich sehr verschieden gesehen werden kann, je nachdem, von welcher Unterscheidung man ausgeht; je nachdem, in welcher Differenz man die Welt verankert. Wenn wir von Welt der Kunst reden werden, ist also immer dieser Begriff gemeint und nicht eine Welt unter anderen. Die Welt der Kunst ist die Welt, gesehen von den Unterscheidungen aus, die die Kunst zur Bezeichnung eines Kunstwerks verwendet.

Diese Begrifflichkeit bringt, ähnlich wie und doch ganz anders als die Zeichentheorie (Semiotik), eine Umlenkung des Interesses von Gegenständen auf Relationen, von Einheiten auf Differenzen auf den Weg, deren theoretische Konsequenzen trotz vielseitiger Bemühungen in den letzten Jahrzehnten noch keineswegs abzusehen sind. Im Folgenden soll nur ein kleiner Ausschnitt des damit angerührten Fragenkreises behandelt werden. Wir gehen davon aus, dass Wahrnehmen ohnehin im Vergleich zum Denken eine hohe Kapazität des simultanen Prozessierens von Unterscheidungen hat und fragen, wie diese Fähigkeit benutzt, verdichtet, gesteigert und mit Hilfe von realen oder imaginären Objekten in Kommunikation übersetzt werden kann. Wir interessieren uns, mit anderen Worten, für eine Theorie der Ästhetik.

10 Die entsprechende Bezeichnung bei Günther $(1978,290)$ lautet: „,compound-contexturality“. 


\section{III.}

Während Denken, sofern es sich an Sprache orientiert, zwangsläufig sequentiell abgewickelt werden muss (weil dies die Struktur ist, die Sprache im Hinblick auf Kommunikation voraussetzt), ist Wahrnehmung (oder im Falle der Imagination: Anschauung) auf Grund von Vorleistungen des neurophysiologischen Systems im bestimmten Umfange fähig, Unterscheidungen simultan zu handhaben. Diese Simultaneität ist ein Bewusstseinsfaktum. Es kann also ganz offen bleiben, ob und wie weit und im welchen Minimalzeiteinheiten auch das neurophysiologische System Informationen simultan prozessiert und dabei zur Informationsgewinnung verschiedene Unterscheidungen gleichzeitig nebeneinander verwendet. Als Ergebnis können wir jedenfalls in einem sehen, dass ein Baum ungewöhnlich groß (nicht klein), weit weg (nicht nah), dunkel (nicht hell) und ohne (nicht mit) Laub an einer bestimmten Stelle (und nicht woanders) steht. Wir sehen ihn auch, aber keineswegs nur, als Typus, als Exemplar der Gegenstandsart Baum, und das heißt: wir sehen ihn im Kontext der Unterscheidung anwesend/abwesend als Repräsentant abwesender Bäume. ${ }^{11}$ Dank der Typifikation haben wir mithin immer schon gelernt, zu sehen, was wir nicht sehen; und es ist gegenüber dieser alltäglichen Selbstverständlichkeit ein schwieriger Prozess (bei dem Philosophie, aber auch Kunst helfen kann), dies wieder zu verlernen und zu lernen zu sehen, dass man nicht sieht, was man nicht sieht.

Wahrnehmungsobjekte entstehen polykontextural, das heißt: durch Optionen in verschiedenen Unterscheidungen zugleich bestimmt. Diese Polykontexturalität wird im Normalfalle durch Typisierungen organisiert, das heißt dadurch, dass viele Unterscheidungen Merkmale suggerieren, die von abwesenden Exemplaren des Typus her bekannt sind. Bäume haben Wurzeln, Häuser haben (außer in Holland) Keller, die aber konkret nicht sichtbar sind. Beide haben unter dem Boden auf unterschiedliche Weise etwas, was sich von dem, was sich sonst da findet, von Erde (bzw. in Holland: Wasser) unterscheidet. Wenn Objekte beson-

11 Dass der Begriff des Typus in dieser Weise auf die Unterscheidung anwesend/abwesend projiziert werden kann, also die Repräsentation des Abwesenden im Anwesenden bezeichnet, ist natürlich eine begriffliche Präzisierung des Wortgebrauchs. Die „typische“ phänomenologische Analyse von Typen (vgl. insb. Husserl 1948, 398 ff.) könnte dadurch an Präzision gewinnen. Denn die „typisch“ an Wahrnehmung orientierte Phänomenologie bringt am Typus neben der unausgesprochenen Leitunterscheidung anwesend/abwesend immer zugleich auch die Mehrheit zugleich charakterisierender Unterscheidungen zur Sprache. Mit dieser hierarchischen Architektonik von Leitunterscheidung und anderen Unterscheidungen kann der Typus Abwesendes ins Anwesende transportieren und damit Unsichtbares sichtbar machen. Da man weiß, um Husserls Beispiel zu nehmen, dass Hunde Gebisse haben, sieht man am anwesenden Hund das Gebiss, auch wenn er es nicht zeigt. Man sieht: er könnte beißen. 
ders auffallen, besonders merkwürdig „sind“, dann liegt das nicht an ihnen, sondern an den Unterscheidungen, die zu ihrer Bestimmung verwendet werde: an den verschieden kontextierten anderen Möglichkeiten, in Bezug auf die es eine Information ist, dass das Objekt so-und-nicht-anders erscheint. Wir sehen den Baum mit Försterblick als krank (also nicht gesund), als zu dicht an anderen Bäumen stehend, als zu fällen usw.

$\mathrm{Zu}$ den Eigentümlichkeiten des Wahrnehmens gehört, dass Unterscheidungen nur benutzt werden können, wenn das Unterschiedene in seinen beiden Momenten, also zum Beispiel als Großes und Kleines, beobachtet werden kann. Die Wahrnehmung erfordert, dass die Unterscheidung als Kontrast auftritt. Das Unterschiedene muss simultan oder im unmittelbaren Nacheinander eines specious present präsent sein, sonst verliert die Unterscheidung ihre Wahrnehmbarkeit. In der Naturwahrnehmung widerfährt es einem denn auch nicht, dass vorherrschende Grüns und Graus als vermisstes Rot erscheinen, und Camille Corot musste infolgedessen, wenn er in seinen Landschaftsbildern diesen Effekt erzielen (wahrnehmbar machen) wollte, irgendwo ein minimisiertes Rot mit sichtbar machen. Wir halten zunächst diese Abhängigkeit von präsenten Kontrasten fest und merken uns die Frage vor, ob und wie die Kunst diese Abhängigkeit sprengen kann.

Man kann solchen Kompaktwahrnehmungen nachdenken, sie analysieren, sie verbal beschreiben und in dieser Form darüber kommunizieren. Man sollte nicht sagen, dass dies nie vollständig möglich sei. Wenn man sich Zeit genug nimmt, lässt sich alles Wichtige auf diese Weise wiedergeben. Damit kann eine für die Wahrnehmung unzugängliche Durchsichtigkeit der operativ wirksamen Unterscheidungen, ein Bewusstwerden und Kommunizieren der das Objekt konstituierenden Kontingenzen erreicht werden. Zugleich wird auf diese Weise alles dem möglichen Zweifel ausgesetzt. Der Zweifel kontrolliert die Kohärenz der Sequenz operativer Informationsverarbeitung und nicht etwa, wie eine lange Tradition gemeint hatte, ihre Realitätsentsprechung, über die er weder positiv noch negativ jemals Auskunft geben könnte. Er greift auch das soeben Wahrgenommene an, lässt sich methodisieren, universalisieren und repräsentiert dann nichts anderes als die Autopoiesis des Systems im System: die Notwendigkeit weiterer und immer weiterer Informationsverarbeitung.

Was dabei verloren geht, ist nicht so sehr (und jedenfalls nicht in einem Sinne, den man bedauern müsste) die „Fülle des Seins““. ${ }^{12}$ Was verloren geht, ist die Simultaneität des Eindrucks. Simultaneisierung ist die einzige Möglichkeit,

12 Die „Fülle des Seins“ ist denn auch nichts anderes als die Aktualität der Ko-präsenz von unterscheidenden Bezeichnungen - und ebenso umgekehrt: „La perception est la pensée de percevoir quand elle est pleine ou actuelle“ (Merleau-Ponty 1964, 49). 
dem Zweifel zu entgehen, der die zeitliche Kohärenz der Informationsverarbeitung kontrolliert und nur mit Vorblick/Rückblick operieren kann. Die Aktualität spricht im Moment für sich selbst. Soweit sie mit Komplexität aufgeladen werden kann, entzieht sie auch das Miterlebte dem Zweifel. Geopfert wird dabei nicht ein Sachwert, sondern ein Zeitwert. Über Sequenzierung kann das Objekt, wenn man sich nur Zeit nimmt, ausreichend identifiziert und beschrieben werden und ausreichend heißt dabei: in dem Umfange, in dem es für weitere Operationen sinnvoll und anschlussfähig ist. Was die Sequenzierung nicht kann, ist: das zu sein, was sie nicht ist, nämlich sequenzlose Simultaneität. Dass die Komplexität des Objekts temporalisiert wird, hat beträchtliche Vorteile - zum Beispiel im Hinblick auf das Verzweigungsvermögen von Anschlussoperationen. Diese Vorteile haben jedoch ihren Preis. Die Evidenz des Zusammenhangs wird herabgesetzt. Das, was in der Einheit der Wahrnehmung oder der Anschauung als zusammengehörig einleuchtet, wird in der Sequenzierung auseinandergezogen. Man muss zum Beispiel entscheiden, womit man die Beschreibung beginnt und hat dann den aus der Entscheidungstheorie bekannten ,anchoring effect"“13: Der Ersteindruck schränkt die Unbefangenheit ein, mit der man weiteres behandelt. Außerdem liegt in der Zeit die Möglichkeit der Unterbrechung, die Chance, dass etwas dazwischenkommt, ${ }^{14}$ die Unsicherheit der Zukunft und die Möglichkeit der Entdeckung von Fehlern. Die Sicherheit des Gesamteindrucks wird in eine Verzögerung der Beurteilung umgewandelt und kann nach dieser Transformation nie wieder erreicht werden. ${ }^{15}$ Auf dem eingeschlagenen Wege der diskursiven Sequenzialisierung kann man zwar auf die Sequenz oder den Diskurs als Einheit reflektieren; man kann den Diskurs rekursiv handhaben, ihn auf sich selbst anwenden, und so verfahren noch die neuesten Techniken operativer Kybernetik. ${ }^{16}$ Damit erzeugt man Realitätsäquivalente - aber man kommt nie wieder zurück zu dem, was man mit dem Kompakteindruck der Wahrnehmung, mit der

13 Vgl. die Varianten: „framing effect“, „topical account“, „,comprehensive account“ (siehe z. B. Kahneman/Tversky 1984).

14 In ihren Rationalitätsimplikationen ist diese Einsicht vor allem von Herbert Simon (1962; 1967) ausgearbeitet worden.

15 Siehe auch Mackay (1964, 177), zu „internal delays“ und daraus folgenden Instabilitäten. Dass dieser Sachverhalt auch positiv genutzt werden kann, zeigt ein von Karl E. Weick $(1985,9)$ verwendetes Beispiel: Ein Polizeibeamter, der einem Verkehrssünder einen Strafzettel ausstellte, wurde von einer sich ansammelnden Menschenmenge bedroht. Er erklärte daraufhin, zur Menge gewandt: „Sie haben soeben der Ausstellung eines Strafzettels durch einen Angehörigen Ihres Oakland Police Departments beigewohnt“. Und während die Umstehenden sich mit diesem Satz beschäftigten, konnte er sich in seinen Wagen retten und davonfahren.

16 Siehe nur von Foerster 1981. 
Simultaneität vieler Unterscheidungen an (ebensfalls nur „konstruierter“) Realität aufgegeben hatte.

All das kann, unter Verzicht auf die Vorteile, vermieden werden, wenn man es bei dem Kompakteindruck der Wahrnehmung belässt. Die Philosophie freilich geriete auf einen grandiosen Irrweg, wollte sie am Falle von Wahrnehmung vorführen, was diese Evidenz ist, mit der sie selbst arbeiten kann. ${ }^{17}$ Auch die Wissenschaft muss diesen Fehler vermeiden. ${ }^{18}$ Philosophie und Wissenschaft können nicht auf Evidenzen, sie müssen auf Unterscheidungen gegründet werden. ${ }^{19}$ Wenn aber Philosophie und Wissenschaft nicht der richtige Weg sind: gibt es einen anderen? Eine Möglichkeit der Aufwertung und Steigerung von Evidenz?

\section{IV.}

Wir erwarten eine solche Aufwertung und Steigerung der wahrnehmbaren, anschaulichen Evidenz von der Kunst. Man hat nicht nur die Wahl zwischen dem factum brutum der komplexen Wahrnehmung und dessen Auflösung durch diskursive Sequenzialisierung mit anschließender Reflexion auf die Einheit des Diskurses. Es gibt einen anderen Weg, eine dritte Möglichkeit: Kunst. Dies ist zunächst eine Mitteilung ohne großen Informationswert. Sie gewinnt, wie wir wissen, ihren Informationswert durch die Unterscheidungen, mit deren Hilfe man bezeichnet, was die Aussage besagt.

Zunächst ist es notwendig, die Beobachtung eines Kunstwerks, sei es während des Herstellungsprozesses, sei es bei einer späteren Wahrnehmung, von den normalen Wahrnehmungen zu unterscheiden. Der Unterschied beruht darauf, dass das Kunstwerk die Unterscheidungen, mit deren Hilfe es sich selbst bezeichnet, anders organisiert und vor allem: auffällig und zirkulär organisiert. Jede Wahrnehmung, jede kleinste Wahrnehmung, auch jede enttäuschende, Erwartungen auslöschende Wahrnehmung inkorporiert das Wahrgenommene in die Welt. Es

17 Husserls selbstsichere (?) Argumentation mit der phänomenologischer Evidenz des SichZeigens war denn auch an eine fatale Überschätzung der kognitiven Leistung des Wahrnehmens gebunden, hatte aber eben dadurch die Struktureigenart der wahrnehmenden Kognition in verdienstvoller Weise erhellt. Den Ertrag hat insbesondere Merleau-Ponty sichtbar gemacht. Bei Merleau-Ponty ist jedoch klargestellt, dass auch die Philosophie nie zur Unmittelbarkeit des Evidenzerlebens zurückkehren kann. „Elle est retour sur soi et sur toutes choses, mais non pas retour à un immédiat, qui s'éloigne à mesure qu'elle veut l'approcher et s'y fondre“ (1964, 161). 18 Ein eher kurioses Beispiel bietet das seinerzeit viel verspottete Argumentieren der Physiokraten mit „Evidenzen“ - bei Le Mercier de La Rivière (1767) nahezu auf jeder Seite.

19 Für die Phänomenologie hierzu eindrucksvoll Waldenfels 1979. 
findet seinen Sinn nur über eine Kohärenzvermutung, die mit wahrgenommen wird - auch und gerade bei überraschenden Wahrnehmungen, die anders gar nicht überraschen könnten. ${ }^{20}$ Das gilt für jede Wahrnehmung, auch für die Wahrnehmung von Kunstwerken. Darüber hinaus erbringen jedoch Kunstwerke eine Mehrleistung, die wir uns genauer ansehen müssen.

Auch hierfür ist der Ausgangspunkt, dass alle bezeichnenden Bestimmungen ihren Sinn durch eine Unterscheidung gewinnen, in der sie die eine und nicht die andere Seite meinen. In der Wahrnehmung ist die Beobachtung einer Unterscheidung an das Auftreten eines Kontrastes gebunden. Das gilt, soweit sie Wahrnehmungen benutzt, auch für die Kunst. Die Kunst ist jedoch freier, den Kontrast selbst für weitere Zwecke einzusetzen. Sie kann ihn zur Regulierung von Anschlussfähigkeit benutzen. Es erscheinen dann zwar beide Seiten der Unterscheidung, aber die eine ist nur dazu da, um die Bezeichnung der anderen zu ermöglichen - so wie der Herr nicht Herr sein könnte ohne den Diener und ohne die Möglichkeit des „crossing“ von Handlungen, die geschehen müssen, ohne dass der Herr sie vollziehen könnte, auf die Seite des Dieners. Die Unterscheidung muss also, um ihre Funktion der Dirigierung der Bezeichnung, auf die es eigentlich ankommt, erfüllen zu können, scharf asymmetrisiert werden, damit der Beobachter den Intentionen des Kunstwerks folgen kann. Das Kunstwerk fügt sich dem Wahrnehmungsgesetz der Simultaneität, missbraucht es aber gleichsam für eigene Zwecke. Es benutzt die Unterscheidung, um die eine und nicht die andere Seite zu fokussieren, stark zu machen und als Punkt für die Anknüpfung anderer Unterscheidungen zu verwenden: der Herr hat ein Schicksal, der Diener hat bloß Glück. Auf diese Weise können über Akzentsetzungen Erwartungen aufgebaut und durchkreuzt werden; über die bloße Kontrastierung hinausgehend kann das Kunstwerk eine exzeptionelle Ordnung sichtbar machen - und sei es nur, wie im Don Quijote, um ihre Brüchigkeit erscheinen zu lassen.

In diesen Möglichkeiten der Akzentuierung dürften auch die Voraussetzungen dafür liegen, dass das Kunstwerk eine Vielzahl von Unterscheidungen kombinieren und über Bezeichnungen der einen und nicht der anderen Seite integrieren kann. Die Unterscheidungen, mit deren Hilfe ein Kunstwerk sich selbst bezeichnet, treten dabei in ein zirkuläres Verhältnis. Jede Unterscheidung profitiert davon, dass andere möglich und angebracht sind. Jede Unterscheidung parasitiert an den anderen. Jede Unterscheidung akzeptiert (und nicht: rejiziert!) die anderen. ${ }^{21}$ Dadurch wird die Willkür des „draw a distinction“ (Spencer

20 Vgl. dazu die Ausführungen über „dés-illusions“ im Anschluss an Husserl bei Merleau-Ponty 1964, $62 \mathrm{ff}$.

21 Hier sei erneut an die „tiefere Zweiwertigkeit“ von Rejektions- und Akzeptationswerten im Sinne Gotthard Günthers erinnert. 
Brown) reduziert. Das Unterscheiden bekommt einen Doppelsinn: es ermöglicht zunächst die bestimmende Bezeichnung der einen Seite (groß und nicht klein, weich und nicht hart, diffus und nicht deutlich abgegrenzt) und setzt sich als Unterscheidung zugleich in eine Beziehung zu anderen Unterscheidungen. Das Hinzufügen weiterer Unterscheidungen im Prozess der Bestimmung des Kunstwerks wird mehr und mehr redundant; was noch zu tun ist oder noch zu erkennen bleibt, erweist sich als bereits vorbereitet. Dies lässt sich aber nicht als Netz von Punkt-für-Punkt-Beziehungen zwischen bloßen Fakten begreifen, und die Kontingenz des Kunstwerks liegt nicht nur in seinem Hergestelltsein. Man versteht den inneren Reichtum eines Kunstwerks daher nicht, wenn man nur das Hergestellte zur Kenntnis nimmt, nur ein bestimmtes quale als quale sieht. ${ }^{22}$ Ein Kunstwerk ist inkorporierte Selektion. Es besteht aus dem, was es zeigt, und aus dem, wogegen das Gezeigte sich absetzt. Die andere Seite der Unterscheidungen, die vermieden wird, wird nicht etwa ausgelöscht, sondern mitangezeigt - so als ob es gälte, die Einseitigkeit der Bezeichnung zu kompensieren; und dies gilt, wohlgemerkt, nicht nur für die gedankliche Rekonstruktion, sondern für die unmittelbare Wahrnehmung selbst. So ist das Kunstwerk in all seinen konkreten Bestimmungen durchsetzt mit „Antimaterie“, nämlich mit dem jeweils anderen, wogegen die das Kunstwerk bezeichnenden Bestimmungen sich entschieden haben. Die Ordnung des Kunstwerks präsentiert daher immer auch invisibilisiertes Chaos, gegen das sie Gestalt gewinnt, und sie kann nur so als Kunst erlebt werden.

In die Sprache der Kybernetik übersetzt heißt dies, dass Kunstwerke unregulierbar sind. Sie besitzen kein ,inneres Modell“ ihrer selbst, an dem sich Herstellung oder Beobachtung orientieren könnten. ${ }^{23}$ Sie realisieren vielmehr den kybernetischen Grenzfall, indem das konkrete Objekt das einzig-mögliche Modell seiner selbst ist. Eben deshalb kann das Kunstwerk Einzigartigkeit beanspruchen. Zweifellos kann sich der Künstler bei der Herstellung einen Plan machen und ein Beobachter wird Strukturen, Stil, Machart usw. erkennen können. Aber es gibt für Reduktion und Aufbau von Komplexität keine Einheitsregel, kein Modemonopol, sondern allenfalls konkurrierende Komplexitätsbeschreibungen, also eine Mehrheit von Vereinfachungsmöglichkeiten. Oder wenn man vorsichtiger formulieren will: in dem Maße, als dies der Fall ist, stimuliert das Kunstwerk Kommunikation.

22 Als Beobachtungstechnik wird deshalb auch eine Art indirektes, absichtsloses Sehen, ein Halbhinsehen oder ein bloßes Mitsehen des Wesentlichen empfohlen (vgl. dazu Böhringer 1985). Dem ist jedoch hinzuzufügen, dass eine solche Ambiguisierung ihrerseits schwer rezeptiert werden kann und dass in ihrer eigenen Anstrengung sich verbirgt, dass jede Unterscheidung „eigentlich“ zur Entscheidung zwingt, was man herstellen und beobachten will und was nicht. 23 Im Sinne von Conant/Ashby 1970. 
Dies führt dazu, dass die Kunst den Beobachter zugleich auf Distanz bringt und unter Ansprüche stellt. Anders als bei der Normalwahrnehmung ist deshalb bei der Kunstwahrnehmung mitbewusst, dass der Gegenstand für die Beobachtung geschaffen ist und sie voraussetzt. Der Beobachter ist aufgefordert, das und das heißt nicht zuletzt: sich selbst als Beobachter - zu reflektieren. Er muss, anders als beim bloßen Wahrnehmen, sehen können, was nicht zu sehen ist. Um dies zu ermöglichen, werden Begrenzungen und Entlastungen benötigt. Das ringsherum Wahrnehmbare wird an Interferenz gehindert. Es rivalisiert nicht mit den Unterscheidungen und Bezeichnungen, die das Kunstwerk konstituieren; und wenn das Kunstwerk mit der Welt ein Gespräch sucht, dann von sich aus. Kunstwerke arrangieren ihr Hier-und-nicht-woanders, ihr So-und-nicht-anders, ihr Dies-mit-dem und nicht mit anderem auf eine Weise, die Kohärenzen verdichtet und Inkohärenzen externalisiert. Die Überraschung besteht dann darin, dass dies trotz des Kohärenzbruchs in der Welt möglich ist. Die Welt, die damit als trotzdem gegeben, als gerade dadurch gegeben zur Evidenz kommt, erhält damit einen neuen, einen überinkohärenten Sinn. Sie ist unvermeidbares Korrelat des Zusammenhangs von Unterscheidungen. Sie wird daher, wenn es gelingt, Zusammenhänge von Unterscheidungen zu verdichten und Kohärenzbrüche an die Grenzen des Kunstwerks zu verlegen, gezwungen, ihren Sinn zu revidieren. Das Kunstwerk nutzt, mit anderen Worten, das zwangsläufige Mitgegebensein von Welt, um zu erzwingen, dass die Welt auch noch dies leistet: die Differenz von artifizieller Verdichtung und Kontinuitätsbruch eines Verweisungszusammenhanges zu überbrücken. Im natürlichen Wahrnehmen könnte dies nur hin und wieder, nur zufällig geschehen im Sich-Ereignen einer Sequenz von Schreck und Erleichterung, von Überraschung und Wiederherstellung der Erwartungsmöglichkeiten. Das Kunstwerk ist als solches erstaunlich.

Diese Erstaunlichkeit wird seit dem 17. Jahrhundert vorwiegend in der Zeitdimension als „Neuheit“ des Kunstwerks interpretiert: als Überraschung durch etwas, was vorher nicht da war. Diese Interpretation lässt jedoch alle ästhetischen und, wie wir gleich sehen, alle logischen Probleme der Kunst ungeklärt. Neuigkeiten bringt die Zeitung jeden Morgen. Wie und weshalb soll die Kunst damit konkurrieren?

Sachlich beruht die Erstaunlichkeit des Kunstwerks auf der Kombination der es charakterisierenden Unterscheidungen. Zum Beispiel ist dasselbe Grün in einem Bild ein anderes Grün, wenn das Bild ihm sagt, es habe an einer bestimmten Stelle ein Nicht-Gelb zu sein und vielleicht zugleich, aber in anderer Richtung, auch noch ein Nicht-Blau, keinesfalls aber ein Nicht-Rot. Die Unterscheidungen, die das Grün einfärben, müssen dann auseinandergehalten werden (etwa mit Hilfe der Unterscheidung von Farben und räumlichen Platzierungen) und sie müssen außerdem auch unterschieden werden von den Unterscheidungen, 
die nicht verwendet werden. Jede dieser Unterscheidungen kommt aber durch Ausschluss von dritten Möglichkeiten zustande. Wie lässt sie sich dann mit dem, was sie ausschließt, kombinieren? Kombination heißt ja offensichtlich mehr als bloße Addition; die eine Unterscheidung soll die andere, obwohl - und vielleicht gerade weil! - sie sie ausschließt, bereichern. So mag zum Beispiel ein Kunstwerk ein anderes zitieren, um mit der Differenz zu spielen. Zitieren ist erst möglich, seitdem Kopieren verboten worden ist. Es geht um das Gegenteil von Imitation, nicht um Identität des Musters, sondern um Differenz der Kunstwerke. ${ }^{24}$ Deshalb wird der Bezug auf das andere Werk oder auf sonstige externe Sinnquellen (etwa: gesellschaftliche Normalverständigungen) als Formproblem im eigenen Werk aufgefasst und so als Fremdkörper assimiliert. In dem Kunstwerk, das zitiert, muss das Zitat einen Formunterschied ausmachen und das Zitat muss, wenn es erkennbar bleiben soll, eine hinreichende Eigenständigkeit aufweisen. Dass es einen Formunterschied ausmacht, liegt jedoch nur an dem zitierenden Kunstwerk. Dieser Unterschied ist also unabhängig vom Zitiersinn des Zitats und doch zugleich abhängig davon, dass das Zitat sich selbst nicht allein dem Zitierenden verdankt. Nicht davon abhängt und auf andere Weise doch davon abhängt - wie ist das möglich? Wie kann das Kunstwerk das auf diese Weise eingeschlossene ausgeschlossene Dritte validieren?

Wollte man diese Frage in logische Begrifflichkeit überspielen, könnte man sagen, dass jede Unterscheidung als Akzeptionswert für andere Unterscheidungen dient; und unter Akzeptionswert wäre dabei mit Gotthard Günther die negierte Rejektion der Unterscheidung zu verstehen. ${ }^{25}$ Die Möglichkeit, dass eine bestimmte Formentscheidung wegen der Unterscheidung, die sie impliziert, nicht passt, muss erwogen und verworfen werden. Die Unterscheidung muss im Hinblick darauf, was sie als ausgeschlossenes Drittes anderer Unterscheidungen diesen zu sagen hat, akzeptierbar sein. Wenn diese Ebene der Akzeption/Rejektion von Unterscheidungen die das Kunstwerk charakterisierenden Festlegungen (Bezeichnungen) kontrolliert, wird, wie Günther zeigt, jede Selektion zur Frage der Akzeption/Rejektion von Unterscheidungen im System; ${ }^{26}$ und dies bedeutet im Effekt, dass es sich um ein unter den Gesichtspunkten der Logik geschlossenes System handelt.

Damit ist jedoch der logische Raum der Möglichkeiten schon bei wenigen Unterscheidungen derart riesig, dass sich die Anfertigung von Kunstwerken nicht rezeptieren lässt. Der Künstler muss es probieren. Er muss in einem rekursiven Verfahren Unterscheidung auf Unterscheidung anwenden und muss sich dabei

24 Für ein klärendes Gespräch hierüber danke ich Hann Trier.

25 Siehe Günther 1976a, 229 ff., 238 ff.; ferner 1976b, 287 ff.

26 Vgl. Günther 1976a, 231 f.; ferner 1976c, insb. Fig. 5 (337). 
durch das, was er schon sieht, führen lassen. Die Unmöglichkeit der Übersicht über die Möglichkeiten wird, wie beim Schachspiel, durch die Geschichte des Spiels ersetzt. Kein Anfang garantiert, wenn man nur nach den Regeln der Kunst verfährt, in sich selbst schon ein gutes Ende. Aber die verbleibenden Entscheidungsfreiheiten sind doch groß genug, dass der Erfolg nicht schlechthin unwahrscheinlich wird - jedenfalls in den Kunstarten, die sich in einem Prozess evolutionärer Auslese gebildet und erhalten haben. Das Resultat ist, wenn es gelingt, das heißt: wenn die Wahrnehmung es validiert, erstaunlich.

Die Erstaunlichkeit des Kunstwerks liegt demnach nicht (bzw. nur für die Wahrnehmung selbst) in der Eigenart des Dinges. Sie liegt in der Art, wie das Abweisen andere Möglichkeiten durch die Kombination der Unterscheidungen, die diese Möglichkeiten anzeigen, ermöglicht wird. Ein Kunstwerk entsteht durch Verdichtung des Zusammenhangs von Unterscheidungen, wobei bestimmte Unterscheidungen verstärkt, triviale Unterscheidungen weggelassen und bedeutende hinzugefügt werden können. Es können Einzelunterscheidungen exaltiert werden - so wenn man einundzwanzig millimetergroße Elefanten in einem ausgehöhlten Nusskern findet und sich an die Relativität aller Größenurteile erinnert fühlt. Es kann aber auch der kombinatorische Reichtum von Unterscheidungen vorgeführt werden - etwa an einer Barockfassade. Ein literarischer Charakter kann ausgeführt sein als ein moralisches Exemplum mit einer nur eindimensionalen Unterscheidung, die im Fall des Exempels Extrembelastungen zu tragen hat; oder er kann mit Ambiguitäten und Widersprüchen angefüllt werden und dadurch eine gewisse Sicherheit gegen moralische Beurteilungen/Verurteilungen bieten, was es erleichtert, sich mit ihm zu identifizieren. ${ }^{27}$ Das Kunstwerk bildet dann den Intersektionspunkt einer Vielzahl von Unterscheidungen, ${ }^{28}$ die anders nie oder nicht so aufeinander bezogen werden könnten.

Besondere Aufmerksamkeit verdient ferner die Technik der Ambiguisierung. ${ }^{29}$ Eine Unterscheidung wird so übernommen und eingebaut, dass sie an der Evidenz der Simultaneität partizipiert, also als notwendig erscheint, und zugleich wird sie so gehandhabt, dass das Kunstwerk sich innerhalb der Unterscheidung nicht entscheidet - dass es weder die eine noch die andere Seite oder beide zugleich bezeichnet. Die Unterscheidung wird dann als Träger einer wei-

27 Vgl., dies nun wiederum unterscheidend als „flat“ und „round characters“, Forster 1974.

28 Dieser Begriff im Kontext von Polykontexturalität bei Gotthard Günther (1978, 289, 291) und in fast gleichem Theoriezusammenhang bei Merleau-Ponty $(1964,114)$.

29 Darauf spezialisiert sind zum Beispiel die Radierungen von Escher, aber auch viele Romancharaktere, etwa solche, die Aufrichtigkeit in der Unaufrichtigkeit suchen (der Comte de Versac in Claude Crébillons „Les égarements du coeur et de l'esprit“ (1961)) oder Sühne in der Schuld. 
teren Unterscheidung ge-/missbraucht: sie wird symbolisch oder diabolisch gehandhabt - oder lässt auch dies noch in der Schwebe. ${ }^{30}$

Dies alles soll der Begriff des Kunstwerks übergreifen - und er ist so weit gebildet im Interesse der Möglichkeit, Kunst $\mathrm{zu}$ unterscheiden von anderen Formen wahrnehmbarer Gegenständen, seien sie nun natürlich oder nur dank Herstellung vorhanden. In allen Fällen, die Anspruch erheben, als Kunst zu gelten, muss die Simultaneität des Wahrnehmens beibehalten und in den für sie typischen Möglichkeiten genutzt werden. Das gilt auch, und in besonderem Maße für Musik; denn auch ein Werk der Musik, ja selbst eine Melodie, ist nur möglich, wenn in der Aktualität, obwohl sie verklingt, eine Mehrheit von ,interessanten“ Differenzen verankert werden, sei es zu den gerade verklungenen und zu den zu erwartenden Klängen, sei es zu gleichzeitigen Klängen anderer Art. Die Anreicherung des allein wahrnehmungsaktuellen Moments läuft auch hier über Unterscheidungen, und die Zeit wird überdies benutzt, um Unterscheidungen aus Unterscheidungen hervorgehen $\mathrm{zu}$ lassen.

Der kaum mehr zu überbietende Grenzfall aber ist der Tanz. Er erfordert angespannteste Aufmerksamkeit der Wahrnehmung, indem er ihr einen Gegenstand anbietet - nur um ihr das Entschwinden ihres Gegenstandes vorzuführen. Auch der Tanz stützt sich auf die Simultaneität des Wahrnehmbaren, aber nur, um es der Wahrnehmung zu entziehen. In dem Maße, als die Wahrnehmung gefordert und minimiert wird, wird zugleich, und erst recht, eine sequenzierende Beschreibung aussichtslos. Der Tanz selbst ist die Sequenz, und er duldet keine andere neben sich. Alles Erkennen wird zum Erkennen des Soebengewesenseins; zu erkennen bleibt nur, was nicht ist. Es entzieht sich dem Schematismus von Sein oder Nichtsein, von wahr oder falsch in einer unbezweifelbaren Aktualität. ${ }^{31}$ Und gerade dies wird so präsentiert, dass es nur im Unterscheidungsreichtum des Moments erfasst werden kann.

Man könnte sich schließlich fragen, ob all dies auch für Literatur gilt, da die Leser ja nicht gleichzeitig lesen. Gleichwohl finden auch die Leser sich in einer gleichen Zeitdistanz zur Textgeschichte. Diese läuft, selbst wenn mit Daten versehen, nicht in ihrer Zeit ab. Sie läuft nicht weiter, wenn die Lektüre unterbrochen wird. An die Stelle der sozialen Simultaneität tritt die zeitunabhängige Jederzeitigkeit des Zugriffs, und diese ist für alle Leser dieselbe. Sie befreit zu ähnlich distanzierten Beobachtungsmöglichkeiten und daher zu Möglichkeiten einer Kommunikation, die die Zeit der Textgeschichte nicht verbraucht und nicht in sie interveniert. Auch auf diese Weise kann also soziale Kommunikation ein Kunst-

30 Ein Beispiel hierfür: Stockton 1969.

31 Der Tanz bringt damit auch die „sokratische“ Beobachtungsweise an ihre Grenze - in Paul Valérys Dialogue „Lâme et la danse“ (1931). 
werk der Literatur so behandeln, als ob es ihr simultan vorläge, und sie kann auch hier von einem Reichtum gesehener und mitgesehener Unterscheidungen ausgehen, die eben deshalb zur Kommunikation reizen, weil jeder Teilnehmer einen anderen Einstiegspunkt wählen kann.

Kunst benutzt mithin die Beweiskraft der Intersektionspunkte von Unterscheidungen, um andere, nicht von selbst sich einstellende Möglichkeiten zu zeigen. Anders als Philosophie und Wissenschaft löst sie das Vorhandene nicht in Sequenzen auf. Sie gestaltet es um. Sie sequenziert nicht, sie simultaneisiert. Obwohl der Arbeitsgang des Herstellens eines Kunstwerks manuelle, gedankliche und eventuell kommunikative ${ }^{32}$ Sequenzen erfordert, muss das Ergebnis auf einmal da sein. Und schon der Arbeitsgang selbst beruht, wenn man so sagen darf, auf der Überzeugungskraft von Zwischenevidenzen. Man kontrolliert ihn durch die Wahrnehmung. Man sieht (oder hört): so weit, so gut. Das gilt auch für literarisch-fiktionale Kunstwerke. Sie müssen sich in der Imagination bewähren, in einer gedanklich simulierten Wahrnehmung; und auch hier kommt es ausschlaggebend auf Klang und Rhythmus der Sprache an, die, gleichsam wie ein Ornament, in sich selbst und im Heranführen anderer Unterscheidungen stimmen muss.

\section{V.}

Unterscheidungen und Bezeichnungen sind Momente einer einzigen Operation, die wir Beobachten genannt haben. Das Beobachten operiert im Kontext seiner jeweiligen Unterscheidung und schließt sich selbst damit als Drittes aus. Es erzeugt den bekannten „blinden Fleck“. Der Beobachter nimmt sich selbst nicht wahr, wenn er beobachtet. „Der Beobachter ist das Nicht-Beobachtbare“.33 Dieses „nicht“ ist nichts weiter als nichts, es ist weder ein Standort außerhalb der Dinge noch eine besondere ontologische Region, die man als „Subjekt“ bezeichnen und zur Begründung der Beobachtung hinterfragen könnte. Es ist nichts, was die Beobachtung ermöglicht. Es ist nichts, was der Beobachtung fehlt. Es ist nichts

32 Die besonderen Probleme, die auftreten, wenn nicht nur manuelle und gedankliche, sondern auch kommunikative Sequenzen erforderlich sind, könnte man am Falle der Herstellung einer Theaterinszenierung studieren.

33 „Zumindest“, heißt es weiter, ,ist es nötig, daß er auf der Kette des Beobachtbaren der letzte sei. Wenn jemand an seine Stelle tritt, wird er zum Beobachteten. Er ist also in der Position des Parasiten“ (Serres 1981, 365). Dass er in der Position des Parasiten ist, heißt dabei nichts anderes, als dass er von der Möglichkeit einer Unterscheidung profitiert, an der er selbst nicht teilnimmt. 
weiter als die Wahl einer Unterscheidung, die es ausschließt, dass die Unterscheidung sich von sich selbst unterscheidet - es sei denn, mit Hilfe der Wahl einer anderen Unterscheidung, für die dann wiederum das Gleiche gilt. ${ }^{34}$

Die onto-logisch inspirierte Metaphysik hatte dieses Problem mit einer letzten, nicht mehr überbietbaren Unterscheidung formuliert: mit der Unterscheidung von Sein und Nichtsein. Diese Unterscheidung wurde zum Kontext der Philosophie, zum Kontext der kategorialen Dekomposition des Seins, zum Kontext schließlich der Reflexionsphilosophie. Wie in einer Art Emanationstheorie versuchte man, aus der Unterscheidung mehr zu gewinnen als die bloße Unterscheidung selbst, wobei das Subjekt (das Denken, das Bewusstsein, die Selbstreferenz) den Platz einnehmen musste, von dem aus die Unterscheidung ihrer eigenen Einheit gegenübertrat.

Wir können eine derart komplexe und historisch reiche (und doch relativ strukturarme) Architektur hier nicht angemessen beurteilen. Es fällt jedoch auf, dass die Theorie selbstreferentieller, beobachtungsfähiger empirischer Systeme die Probleme nicht mehr in dieser klassischen Weise stellt und deshalb auch zu anderen Vorstellungen über Problemlösungen kommt. ${ }^{35}$ Die simple, aber folgenreiche Reformulierung liegt in der Einsicht (die ihrerseits keineswegs neu ist, aber durch den unterscheidungsbezogenen Beobachtungsbegriff einen neuen Sinn erhält), dass ein Beobachten von Beobachtungen möglich ist.

Was daraufhin Beobachtung zweiter Ordnung oder Kybernetik zweiter Ordnung genannt wird, kann sich, je nach Ausstattung der Systeme mit Selbstreferenz, auf Fremdbeobachtung und auf Selbstbeobachtung beziehen. Entscheidend ist: dass die Beobachtung einer Beobachtung diese in einen Kontext versetzen kann, der der beobachteten Beobachtung selbst unzugänglich ist. Ja man kann sogar einen Sport daraus machen, eine Beobachtung oder einen Beobachter im Hinblick auf die Unterscheidung für ihn zugänglich/für ihn unzugänglich zu beobachten. Die Faszination durch gerade diese Unterscheidung beruht darauf, dass sie nur in der Fremdbeobachtung gehandhabt werden kann, also eine genuin soziale Komponente hat, und bei der Anwendung auf Selbstbeobachtung zerplatzt. Eine gut zweihundertjährige kulturelle Tradition solcher Beobach-

34 In dieser Einsicht konvergieren Analysen, die von einer Radikalisierung ontologischer Fragestellungen ausgehen - siehe im Anschluss an Sartre insb. Merleau-Ponty 1964, 77 ff. - mit der schlichter formulierten Einsicht des Physikers, dass man nicht sieht, was man nicht sieht und dass dies ein internes, nicht ein externes Problem beobachtender Systeme ist (von Foerster 1979). 35 Dass mit dieser Kontrastierung Konvergenzen nicht ausgeschlossen sein sollen, ist in der vorigen Anmerkung angedeutet. An anderer Stelle (Luhmann 1986a) habe ich versucht, die Husserl'sche Epoché als Einführung eines Rejektionswertes (Günther) in die Ontologie zu interpretieren. 
tungsschemata - für die Literatur und die Psychologie: bewusst/unbewusst; für die Soziologie zumeist: manifest/latent - zeigt, dass dies heute nahezu routinemäßig möglich ist und zu den vielseitig applaudierten Techniken der Aufklärung, der Entlarvung und der Therapie gehört. ${ }^{36}$

Mit etwas anderen Begriffen kann man auch sagen: die einfache Beobachtung setzt sich zu ihrem Gegenstand in ein symmetrisches Verhältnis. Sie ist, was sie sieht, und sie ist nichts anderes. Die Beobachtung der Beobachtung muss diese dagegen asymmetrisieren. Sie sieht Bedingungen einer gerichteten, intentionalen Operation. Sie kann daher Eigentümlichkeiten und Resultate der Beobachtung, besondere Sensibilitäten, Wahl des Gegenstandes, Täuschungen und Verzerrungen auf den Beobachter zurechnen. Sie kann die Beobachtung verstehen, wenn sie ihr Augenmerk auf den Beobachter richtet und feststellt, wie er dazu kommt, so und nicht anders zu beobachten. Sie kann die Beobachtung aber auch schlicht duplizieren, nämlich ebenfalls sehen, was der andere sieht. Die Beobachtung der Beobachtung transformiert diese aus einer symmetrischen (die sie für sich bleibt) in eine asymmetrische Relation. Sie hat dann die Wahl, ihre weiteren Operationen an den beobachteten Beobachter oder an seinen Gegenstand anzuknüpfen, immer vorausgesetzt, dass sie Distanz hält und die beobachtete Beobachtung von der Beobachtung der Beobachtung unterscheiden kann. ${ }^{37}$

Angewandt auf unser Thema kann man deshalb fragen: Was beobachtet man, wenn man beobachtet, dass ein anderer ein Kunstwerk beobachtet? Abgesehen von der Frage, was das Kunstwerk „ist“, muss man mit der Möglichkeit rechnen, dass es auf die Möglichkeit des Beobachtens von Beobachtungen hin produziert ist. Die Erstaunlichkeit des Kunstwerks wäre dann nicht nur eine Art Rätsel, das jeder für sich zu entschlüsseln hat, um sich selbst dann mit einem Aha-Effekt zu beruhigen (sobald man den trompe l'oeil durchschaut hat). So wäre kein Anspruch auf Dauer zu begründen. Vielmehr scheint das Kunstwerk als Medium sozialer Kommunikation zu funktionieren und nur deshalb die eigene Erstaunlichkeit zu benötigen.

Wir wiederholen und vertiefen: Das eigene Beobachten ist sich selbst nichts. Man nimmt nur das Wahrgenommene wahr und das ändert sich für das eigene Wahrnehmen im Prinzip nicht, wenn man das Wahrnehmen anderer wahrnimmt. Was beim Wahrnehmen zweiter Ordnung hinzukommt, ist jedoch Einsicht in die Distanz zwischen Wahrnehmung und Objekt. Man kann so und nur so (und niemals im Fichte-Verfahren aus der Absolutheit des eigenen Ich heraus)

36 Es gibt, soweit ich sehe, keine zureichende Darstellung der Entwicklungsgeschichte dieses Schemas. Ellenberger (1970) bietet reine Fachgeschichtsschreibung; siehe aber van den Berg 1974. 37 Interessante strukturelle Überlegungen dazu, bezogen auf Selbstbeobachtung im subjekttheoretischen Kontext der Wissenschaftslehre Fichtes, bei Baldus 1982, $17 \mathrm{ff}$. 
zwischen Subjekt und Objekt unterscheiden. Man sieht am anderen, dass er als Beobachter nicht nur nichts ist (schon weil man ihn wahrnehmen kann) und schließt daraus auf sich selbst zurück. Der andere nimmt mich wahr als jemanden, der Kunst beobachtet und macht mich für den Sinn, den ich sehe, mitverantwortlich. Nur wir beide können jeden von uns vom Objekt unterscheiden. Mit der daraufhin möglichen Unterscheidung von Objekt und Subjekt hat man ein Zurechnungsproblem und zugleich die Chance, das Problem durch Zurechnung zu lösen. Entweder sieht der andere das Objekt so, weil es so ist; oder er sieht es auf seine Weise, einem selbst die Freiheit lassend, es anders zu sehen. In der Wahrnehmung zweiter Ordnung entsteht mithin Freiheit - so vor allem die Freiheit der verschiedenen und doch objektkoordinierten Körperbewegung in einer dann als Raum erfahrenen überinkohärenten Welt. Könnte es sein, dass das Kunstwerk diese Möglichkeit, eine überinkohärente Welt zu erzeugen, aufgreift, ausbaut und eventuell gegen die Welt richtet, wie wir sie kennen?

Das Kunstwerk zeichnet sich durch ein exzeptionelles, besonders reichhaltiges Zusammenführen von Unterscheidungen aus. Verschiedene Beobachter können daher Verschiedenes sehen und durch diese Verschiedenheit zusammengeführt werden. Zugleich sehen sie dasselbe und erfahren diese Einheit von Identität und Differenz. Schließlich ist diese Koordination abgelöst von natürlichen Gegebenheiten und daher fragwürdig; sie ist frei, irgendeinen Sinn anzunehmen. Das Kunstwerk fordert daher auf, sich über seinen Sinn zu verständigen.

Schon bei ungewöhnlichen Ereignissen - das Licht geht aus, zwei Autos stoßen zusammen, ein Passant fällt zu Boden - ist es, wenn mehrere es sehen, fast unvermeidlich, darüber zu kommunizieren, obwohl doch jeder gesehen hat, was passiert ist. Gerade das Schonverständigtsein im Ungewöhnlichen ist der Anlass, sich an der Kommunikation zu beteiligen und dies wechselseitig zu erwarten. Man würde auffallen, würde man das Auffällige anscheinend ignorieren. Ebenso, und mit dem gleichen Mechanismus, veranlasst das Kunstwerk Kommunikation - sei es verbale Kommunikation, sei es, wenn es gut geht, das stillschweigende Mitteilen des sich Koordiniertwissens im gemeinsamen Sehen oder Hören. Der Kommunikationsanlass liegt auch hier in der offensichtlich werdenden Nichtidentität von Objekt und Beobachter. Man lebt nicht einfach bruchlos in der Kontinuität seines eigenen Beobachtens mit jener normalen Nichtberücksichtigung der eigenen Sichtweise. Man sieht, dass der andere stutzt und sein Beobachten intensiviert; oder man sieht am Objekt, dass der andere und man selbst eigentlich stutzen müssten. Das Kunstwerk befreit zur Kommunikation, indem es die Beobachtungsabhängigkeit seiner Unterscheidungen betont, den Abstand von Objekt und Beobachter als soziale Situation verdeutlicht und jedem einen Eigenbeitrag zumutet. Das für ihn Auffällige ist ja nicht notwendigerweise das, was den anderen auffällt, obwohl das Kunstwerk die Einheit aller Perspektiven behauptet. 
Wie weit eine Verbalisierung der Kommunikation sinnvoll, möglich oder umgekehrt: gerade deplatziert ist, weil sie die Feinsinnigkeit des Kunstwerks nicht erreicht, ist dabei eine weitere Frage. Jedenfalls kann die Rede nie zum „immédiat perdu“ (Merleau-Ponty) zurückkehren. Da die Simultaneität des Wahrnehmens (im Unterschied zur Sequenz des begleitenden Denkens und Redens) den Ausgangspunkt bildet, kann verbale Kommunikation in weitem Umfange eingespart bzw. auf eine Art Spitzenausgleich oder auf bloßes Bestätigungsverhalten beschränkt werden. Das Thema steht fest durch das, was der Wahrnehmung gegeben ist. Das heißt aber auch, dass mehr Konsens und mehr Dissens zugleich möglich sind. Man darf annehmen, dass in Situationen dieses Typs ein Potential für die Ausdifferenzierung besonderer Interessen, besonderer Expertise (vor allem der Künstler selbst) und schließlich eines besonderen Sozialsystems Kunst angelegt ist, das dann seinerseits die Anforderungen an sachkundige Kommunikation steigert.

Kunst ist nach all dem ein besonderes, ein eigenartiges Medium der Kommunikation, und es ist diese Eigenart der Kunst, die im Laufe der Zeit zur Ausdifferenzierung eines darauf spezialisierten Sozialsystems geführt hat. Was immer sonst als „Medium“ geeignet ist: ${ }^{38} \mathrm{im}$ Bereich der Kommunikation entstehen symbolisch generalisierte Medien, wenn immer die Einheit in der Differenz von Ego und Alter unter steigenden Ansprüchen zum Problem wird. Vielleicht ist es zunächst eine Art Kontingenzlust: man möchte etwas sich anders vorstellen, anders herstellen, etwas Abwesendes sichtbar machen oder etwas Anwesendes verschönern und dafür Zustimmung finden können. Die Wahrnehmbarkeit ist dann Garant für den Zugang der anderen, die Erstaunlichkeit garantiert ihre Aufmerksamkeit und die Form versucht jedenfalls, ihre Zustimmung zu gewinnen. Ähnlich wie beim Geld darf man nicht voraussetzen, dass das Medium wie ein Mittel zum Zweck für eine bestimmte Funktion erfunden worden ist. Die Bereicherung der Welt durch Kunst ist nicht etwas, was man sich vorstellen kann, bevor es Kunst gibt. Erst wenn es akzeptables Geld in hinreichendem Umfang (und Arbeitsteilung und hinreichend große Märkte usw.) gibt, kann Geld benutzt werden, um Tauschmöglichkeiten zu bereichern. Und erst wenn Kunst hinreichend geläufig ist, kann man darin eine

38 Für einen sehr abstrakten Begriff des Mediums kann man sich an den Essay „A Paradigm of the Human Condition“ des späten Parsons (1978) wenden. Dort heißt es: „The concept of a medium to us implies that it establishes relations between or among diverse and variant phenomena, tendencies, and so on. If this is the case, media must be able to relate to these entities beyond simply dissolving into their diversity. This property of a medium, namely, its capacity to transcend and thereby relate, diverse things, may be called its generality, which varies by levels of generalization“ (1978, 395). In dem besonderen Bereich der Handlungssysteme müssen für diese Funktion Symbole eingesetzt werden, das heißt Sinnformen, die den Beteiligten die Einheit in der Verschiedenheit von Ego und Alter präsentieren können. 
Möglichkeit sehen, in einem Prozess der „Hyperkorrektion“ von Abweichung durch Abweichung ${ }^{39}$ die Möglichkeiten, die Welt $\mathrm{zu}$ beobachten, $\mathrm{zu}$ erweitern. Und erst dann kann die Kunst eventuell dasjenige Medium werden, das andere Bereiche der Gesellschaft ständig darauf hinweist, wie eng ihre Weltsicht ist.

Da das Kunstwerk simultaneisierte Komplexität anbietet, steht alle soziale Kommunikation über Kunstwerke unter dem Vorzeichen verschiedener Zugangsmöglichkeiten. Die Beobachter sind dann nicht nur durch das Kunstwerk überrascht, sondern auch und erst recht durch die Verschiedenheit ihrer Beobachtungen. Wenn nun dies wiederum beobachtet wird, sucht man zunächst Urteilskriterien, etwa solche des guten Geschmacks. Wenn solche Kriterien angeboten werden, können Kunstwerke sie übernehmen, sich für oder gegen sie entscheiden oder damit zu spielen beginnen. Diesem Prozess kann man sich durch Formulierung „absoluter“ Kriterien nicht entziehen, denn schon deren Formulierung ist paradox und widerlegt den in Anspruch genommenen Sinn. Es bleibt die Möglichkeit, zu diskutieren, ob etwas Kunst ist oder nicht. Es entstehen Abgrenzungsbegriffe wie zum Beispiel Kitsch. In diese Lage gebracht, mag die Kunst sich provoziert fühlen, ihre eigenen Grenzen zu testen und auszuweiten. Analog zu: alles ist käuflich, alles kann zum Rechtsfall werden, alles ist politisierbar, gibt es nun Kunstwerke, die sich darauf beschränken, den Anspruch darzustellen, es zu sein. Dann wird es aber zum Test, ob es gelingt, durch Kunst Kommunikation über Kunst auszulösen. Die Grenzen des Systems liegen in der Faktizität der Kommunikation, und die Extravaganz der Kunst kann schließlich bis zu dem Punkt getrieben werden, an dem die Wahrnehmung von Kunst zum Anlass wird, darüber nicht zu kommunizieren. Im Zusammenfall von Kommunikation und Nichtkommunikation bestätigt sich so der Sinn der Sinnlosigkeit der Welt.

\section{VI.}

Wenn die vorstehenden Überlegungen zutreffen, müsste es möglich sein, Zusammenhänge festzustellen zwischen dem Formangebot der Kunstwerke einerseits und dem Ausmaß, in dem Kunst als soziales System ausdifferenziert ist. So kann man zum Beispiel innerhalb sehr weiter Zeitspannen beobachten, dass eine Erweiterung des Zulässigen mit einer Verschärfung der sozialen Kontrolle korreliert. Einerseits wird mehr möglich, Gewagteres möglich, schwer zu Verstehendes möglich, und andererseits geraten Kunstwerke und Künstler in Abhängigkeit von einem kunstsystemintern geregelten Unterschied von Erfolg und Misserfolg.

39 Speziell hierzu Van Parijs 1977. 
Durch einen ausdifferenzierten Kommunikationszusammenhang getragen, kann das Kunstwerk mehr auf Form setzen als auf Wahrnehmung, das heißt: an die Wahrnehmung nichtselbstverständliche Anforderungen stellen und diese durch Form legitimieren. Damit verlagert sich aber zugleich das Erkennen und Anerkennen von Kunst in Zonen, in denen sowohl Erfolg als auch Misserfolg (und beides nach systemintermen Beurteilungen) möglich sind. Außerdem wird es möglich, dass Kunstwerke erst lange nach ihrer Entstehung als solche wahrgenommen und zum Beispiel aus Dorfkirchen herausgezogen und in Museen überführt werden; und ebenso kann der große Erfolg gewagter Kunst sich erst mit erheblicher Verspätung einstellen. Ein Beispiel ist El Greco.

Solche Entwicklungen lassen sich als evolutionär allmählich zunehmende Ausdifferenzierung eigenständiger Kunstkommunikation an Hand von eigens dafür geschaffenen Objekten begreifen. Man kann aber auch mit neueren Entwicklungen der Evolutionstheorie und der Systemtheorie davon ausgehen, dass es Bruchstellen mit raschen und radikalen Stabilitätsveränderungen gibt, so dass man, zumindest auch, mit diskontinuierlichen Ausdifferenzierungsschüben rechnen muss. In diesem Sinne mag es einen Umschlagspunkt geben, von dem ab ein ausdifferenziertes System ,autopoietisch“ wird, das heißt: seine Strukturen nur noch selbst spezifizieren kann. Das besagt dann auch, dass das System nur noch eigene Unterscheidungen verwenden kann. Solche „Katastrophen“ werden sich in ein breites Vorher/Nachher-Geschehen eingebettet finden, das heißt durch massive Inkonsistenzen vorher und nachher begleitet sein, so dass einem Beobachter die zeitliche Lokalisierung schwer fällt. Und dennoch kann man rückblickend erkennen, dass sie stattgefunden haben, wenn das gesamte System auf autopoietische Selbstreproduktion umgestellt worden ist und Umweltabhängigkeiten, so unvermeidlich sie sind, nur noch als Störung wahrnimmt.

Innerhalb dieses sehr allgemeinen und ausfüllungsbedürftigen Theorierahmens sollen drei Hypothesen etwas genauer formuliert werden. Sie beziehen sich sämtlich auf die Art und Weise, in der es dem Kunstwerk freigestellt wird, Unterscheidungen zu kombinieren.

Die erste Hypothese lautet, dass Kunstwerke bei zunehmender Ausdifferenzierung des Sozialsystems Kunst auf Leistungen der Typisierung mehr und mehr verzichten können, ja schließlich sogar verzichten müssen. Wir befinden uns hier in der Nähe einer Diskussion, die unter Begriffen wie Imitation oder Repräsentation geführt werden, die jedoch beide den Sachverhalt nicht so scharf erfassen wie der phänomenologische Begriff der Typisierung..$^{40}$ Unter Typisierung

40 Nicht so scharf deshalb, weil sowohl Imitation als auch Repräsentation Programmbegriffe der Tradition sind, die sich nicht, oder nur schwer, verwenden lassen, um verständlich zu machen, weshalb man sie aufgibt und was damit verloren bzw. gewonnen wird. 
verstehen wir, wie erinnerlich, eine Anreicherung von Wahrnehmung durch Transport des Abwesenden in das Anwesende. Vielleicht war gerade dies, man denke an die Höhlenmalerei, eine der ältesten Leistungen von Werken, die wir heute als Kunst betrachten. Es handelt sich jedenfalls um eine Anreicherung mit Unterscheidungen. Der Hund blickt treu zu seinem Herrn auf; man sieht, dass er beißen könnte, aber offensichtlich nicht gewillt ist, es zu tun. Dasselbe findet sich auch auf Bildern. Die Kommunikationsanforderungen werden gering gehalten. Beißen/nichtbeißen an einem nichtsichtbaren Gebiss sichtbar zu machen, ist eine schon erstaunliche Leistung - im Leben wie in der Kunst. Oder: Eine Frau bringt ihrem Mann einen soeben eingetroffenen Brief. Dies ist auf Grund koordinierter Wahrnehmungen fast ohne Kommunikation möglich. Wenn der Vorgang als Bild gemalt wird, kann gerade die Kommunikationslosigkeit der Kommunikation wiedergegeben, herausgeholt und verschärft werden. Eine große Zahl von Unterscheidungen wird so ins Bild gebracht: Vertrautheit, die ohne Kommunikation auskommt; der Brief ist gerade eingetroffen, denn man bringt keine Briefe, die schon seit Tagen auf dem Schreibtisch liegen; die Frau bringt ihrem Mann, aber nicht der Mann seiner Frau die gerade eingetroffene Post usw. Auf diese Weise kommen Unterscheidungen ins Bild, die den Wahrnehmungssituationen des täglichen Lebens entsprechen und mittels Typisierung weit über das Kunstwerk hinausgreifen können. Man mag mit leichter Wehmut bedauern, dass solche durch Typisierung bedingten Unterscheidungsverdichtungen aufgegeben worden sind. Man malt nicht mehr so, man dichtet auch nicht mehr so. Der Grund scheint zu sein, dass eine Kommunikation über das so Herangeholte keine Kommunikation über Kunst wäre.

Die zweite Hypothese lautet: dass Kunstwerke bei zunehmender Ausdifferenzierung der Kommunikation über Kunst darauf verzichten können (und vielleicht: darauf verzichten müssen), Normalperspektiven vorauszusetzen, in denen der Beobachter das Kunstwerk zu beobachten und zu beurteilen hat. Die Differenzierung setzt sich auch gegen die sonst allgemeingültigen Wahrnehmungsweisen durch, und das Kunstwerk hat durch das Arrangement seiner eigenen Unterscheidungen und Bezeichnungen mitzuverantworten, dass es nicht in der üblichen Weise beobachtet und beschrieben werden kann. Man kann sich das am Übergang von gegenständlicher Malerei zu abstrakter Malerei verdeutlichen, aber auch an Entwicklungen der Worttextkunst bis hin zur „konkreten Poesie“, also am Verzicht auf einen sprachlich normalen, sinnführenden, bloß „schöneren“ Text zu Gunsten freierer Wortsinn- und Wortklangkombinationen. Auch die Musik beteiligt sich durch Boykottieren von sei es akustischen, sei es konventionellen Garantien der Hörsicherheit. All das macht die Kunst, mehr als zuvor, abhängig von momentaner Wahrnehmung und insofern eigenständiger in ihrer Funktion als Medium der Kommunikation. 
Mit diesen (und vielleicht weiteren) Änderungen der Formansprüche und Erkennbarkeitsvoraussetzungen von Kunst ändert sich zwangsläufig auch das intendierte „Erstaunen“. Es verliert seine Assoziationen mit Schreck, Schauder, Ehrfurcht, Heiligkeit und Hybris; es verliert jene symbolischen Qualitäten, die zur Vermittlung zwischen vertrauten und unvertrauten Bereichen der Welt oder dann zur Darstellung religiöser bzw. politisch-stratifikatorischer Differenzen erforderlich waren. ${ }^{41}$ Erstaunen wird dann zu der Art und Weise, mit der Kunst auf sich selber aufmerksam macht. Dann aber wird die autopoietische Reproduktion des Erstaunlichen zum Problem, und man versucht (in der Hoffnung, niemals an ein Ende zu kommen) dieses Problem durch den Neuheitseffekt des Erstaunlichen, also in der Zeitdimension zu lösen. Die Frage, ob etwas „neu“ ist, kann dann nicht mehr dem nachträglich-vergleichenden Urteil eines externen Beobachters überlassen bleiben; sie leitet die Suchstrategie bei der Anfertigung des Kunstwerks selbst. Das aber heißt: dass die Kunstproduktion reflexiv werden muss als ständig-rekursive Anwendung von Kunst auf Kunst. ${ }^{42}$

Schließlich kann man drittens beobachten, dass auf Grund der erweiterten eigenen Möglichkeiten das Kunstwerk die Welt als einen Kontext behandeln kann, der weder imitatorisch noch typisierend ausgebeutet, aber gleichwohl über Differenz in das Kunstwerk einbezogen wird. Zum Beispiel erhöht, wenn man einmal von einer Unterscheidung von Tönen und Geräuschen ausgehen darf, die moderne Musik ihre Geräuschakzeptanz mit Hilfe eines Formarrangements, das noch als geordnet erfahren werden kann. Die Differenz von Tönen und Geräuschen wird intern nochmals reproduziert, und zwar bis in die Tonqualität hinein, die in einer Richtung harmonisch und in anderer Richtung zugleich disharmonisch gehört werden kann. ${ }^{43}$ Für Textkunstwerke kann man ähnliche Tendenzen

41 Ein wenig spekulativ, aber untersuchenswert ist die Hypothese, dass die Erfindung von Schrift bzw. von Buchdruck diese Entwicklung ermöglicht, wenn nicht ausgelöst hat. Denn durch Schrift und Buchdruck wird die Differenz vertraut/unvertraut ohnehin sozialisiert: der eine kennt diesen, der andere jenen Text, und jeder weiß, dass es Texte gibt, die ihm unvertraut aber anderen vertraut sind. Gegeben diesen Entwicklungsstand des Gesellschaftssystems wird es immer weniger nötig, die Unterscheidung vertraut/unvertraut über Symbolisierung eben dieser Differenz in die Gesellschaft wiedereinzuführen. Hierzu auch Luhmann 1986a.

42 Dies gilt auch, und verstärkt, für eine Art Superreflexion: Wenn Dichter zum Beispiel normale Worte so verwenden, dass ihr ursprünglicher Sinn von allen historischen Abweichungen und Verzerrungen befreit wieder zur Geltung kommt.

43 Frank Rotter $(1985,60)$ bemerkt dazu ganz allgemein, dass „Musik etwas von dem Chaos zitieren muss, um dessen Bannung es [...] geht“. Man kann, daran anschließend, dann den Eindruck haben, dass diese Chaotisierung an die Grenzen dessen getrieben wird, was noch als Ordnung erkennbar bleibt - eine Art Öffnung des Tonkunstwerks gegen eine geräuschhafte Umwelt, die mit der „Tonmalerei“ begonnen hatte. 
zur einer durch Form gehaltenen inneren Chaotisierung feststellen, für bildende Künste das gleiche, so dass sich daraus eine Tendenz erschließen lässt, von der Kunst aus die Welt als ein noch ordnungsfähiges Chaos zu deuten - als ein zumindest ästhetisch noch ordnungsfähiges Chaos, das nichts Imitierenswertes mehr anbietet.

Im Ergebnis führen diese Veränderungen dazu, dass das Einzelkunstwerk nicht mehr beansprucht, ein Monopol für die Modellierung der Welt zu besitzen. Eher eröffnet es verschiedene Zugänge zur Polykontexturalität der Welt und verlagert damit die Weltproblematik in soziale Kommunikation an Hand von Kunst. Der Unterschied soll durch zwei Skizzen verdeutlicht werden.

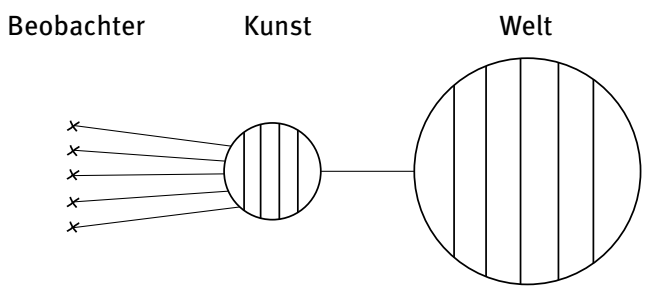

Abb. 1

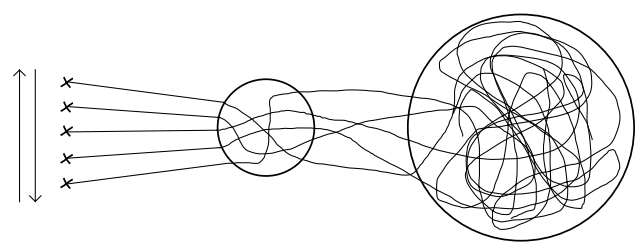

Abb. 2

In der Ausdrucksweise von Gordon Pask (1981) und Stein Bråten (1983) könnte man auch sagen: erst durch Auflösung des „model monopoly“ und durch Einarbeitung von Perspektiveninkongruenz in das Kunstwerk entsteht „consciousness“ an Hand von Notwendigkeiten, über Inkongruenzen zu kommunizieren. Der Weltausgleich wird über das Kunstwerk in die soziale Kommunikation verlagert, wobei die Simultaneität der Unterscheidungen, die im Kunstwerk festgehalten sind, zugleich verhindert, dass die Kommunikation diskursiv abläuft. Herausforderung von Kommunikation also bei Ablehnung von Diskursen - wer es nicht erlebt hat, wird nicht glauben, dass dies möglich sei. Denn die Wirklichkeit wird nur durch die Abbildung 3 adäquat repräsentiert (die ihrerseits noch den Fehler hat, dass sie nicht als sich selbst enthaltend gezeichnet werden kann). 


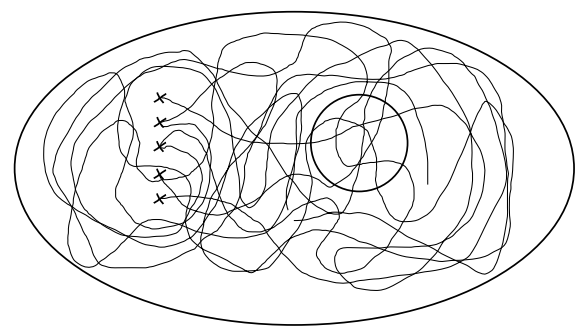

Abb. 3

Über die Beurteilung dieser Entwicklungen kann man sicher verschiedener Meinung sein. Auch wäre zu diskutieren, ob es sich bei solchen Brüchen mit gängigen Beobachtungsweisen nur um unvermeidliche Kosten des Ausnutzens freierer und reicherer oder ungewöhnlicherer Kombinationsmöglichkeiten im Kunstwerk selbst handelt, also um eine bloße Verschärfung und Internalisierung der Differenz zwischen interner Kohärenz und externer Inkohärenz, oder ob die Schockierung und Verabschiedung der Normalperspektive des Betrachters gerade zu den Unterscheidungen gehört, die das Kunstwerk als seine Eigenleistung ansieht und die es, wenn man so sagen darf, selbst für schön hält. Nur in diesem Fall hätte das Kunstwerk jene überinkohärente Welt im Auge, auf die es in der Funktion der Kunst ankommt. Dann müsste die Kunst, ähnlich wie das Geld, die Entfremdung als funktionsnotwendig rechtfertigen können, also hier: an der Form zeigen, dass es so ist. Und es würde nicht genügen, durch die Notwendigkeit, etwas auszuprobieren, etwas anders zu machen, getrieben zu sein.

Ob diese Veränderungen Fortschritt oder Verfall bedeuten, steht hier nicht zur Diskussion. Sie werden hier nur angeführt, um zu belegen, dass es Zusammenhänge zu geben scheint zwischen der Ausdifferenzierung eines besonderen Sozialsystems Kunst und Formentwicklungen bei Objekten, die als Kunst hergestellt und akzeptiert werden. Die Beurteilung bleibt Sache des Kunstsystems (bzw., solange ein solches System nicht ausdifferenziert ist, Sache der Gesellschaft). Auch erklären im strengen Sinne kann man diese Entwicklung nicht, wenn „erklären“ heißen soll: die Ursachen aufzudecken, ohne welche es nicht so gekommen wäre, wie es gekommen ist. Nach heutigen Theorien der Morphogenese und der Evolution sind solche Ursachen in hohem Maße Zufälle. Sie können sich nur dadurch auswirken, dass ein selbstreferentielles System sie als Ursachen akzeptiert und sich genötigt sieht, auf sie zu reagieren. Gerade im Falle der Kunst muss man denn auch in weitem Umfange damit rechnen, dass es Gelegenheiten, historische Koinzidenzen, Einfälle am entstehenden Werk sind, die zu Formerfindungen führen, die Eindruck machen, Stil machen, Epoche machen. Die neuzeitliche Theorie der Kunst war ist seit langem bereit, dies anzuerkennen, ja 
geradezu zu feiern. Sie feiert damit die Kunst als selbstreferentielles System, das bereit und in der Lage ist, mit Materie und mit Zufällen zu arbeiten, weil es sich selbst immer schon voraussetzen kann.

\section{VII.}

In vielen Funktionssystemen haben sich binäre Codierungen bewährt. ${ }^{44}$ Sie verdoppeln die Menge möglichen Sinns, indem sie jedem Item eine Gegenmöglichkeit gegenüberstellen. ${ }^{45}$ Das erzwingt eine Digitalisierung des Sinnbereichs und zwingt damit zu Entscheidungen. Erleben und Handeln in codierten Bereichen ist ständig mit Entscheidungen beschäftigt, es gleitet nicht einfach bruchlos (,,analog“) am intrinsisch bewegten Bewusstsein entlang. Wenn es codierten Entscheidungszwang gibt, entsteht daraus ein Bedarf für Gesichtspunkte, Kriterien, Regeln, Programme, an die man sich halten kann. Größere Entscheidungsmengen müssen zu Systemen zusammengefasst und in ihrer (relativen) Konsistenz kontrolliert werden, weil anderenfalls nur eine Vielfalt ohne eigene Ordnung entstehen würde.

Hinter diesen Überlegungen steckt eine Sachgesetzlichkeit, ${ }^{46}$ die auch für die Welt der Kunst gilt. Damit ist noch nicht gesagt, dass der Kunst zu allen Zeiten (oder im Besonderen: heute) eine Codierung gelingt; wohl aber, dass es weitreichende Folgen haben wird, wenn sie nicht gelingt. Ein Verzicht auf jede Codierung müsste auf einen Verzicht auf Kunst als distinktes Phänomen hinauslaufen. Sie könnte dann immer noch als eine beim Arbeitsamt geführte Kategorie von beruflichen Beschäftigungen dienen, die zum Versagen von Arbeitslosenunterstützung berechtigt; aber sie würde ihre Produkte nicht mehr von anderen Artefakten unterscheiden können. Wenn wir diesen Grenzfall (den anzunehmen

44 Vgl. Luhmann 1986b; 1986c.

45 Das ist denn auch der ursprüngliche Sinn von „Code“: eine Zuordnungsregel zu garantieren, die zwei Zeichenmengen verknüpft. Die Übertragung des Begriffs auf die dadurch digitalisierten Zeichenmengen selbst ist sekundär und sollte vermieden werden.

46 Dass diese „Sachgesetzlichkeit“ als wissenschaftliche Theorie formuliert wird und damit im Bereich des ihrerseits Codierten, also möglicherweise Falschen liegt, soll damit natürlich nicht bestritten sein. Im Gegenteil: gerade das wird mit der These mitbehauptet. Die „Sachgesetzlichkeit“ ist auf sich selbst anwendbar. Man muss allerdings sorgfältig unterscheiden, ob man sie selbst bestreiten will, was sehr weittragende Folgen für eben dieses Bestreiten hätte, oder ob man nur in Zweifel ziehen will, ob die Kunst schlechthin oder ob nur die heutige Kunst sich dem fügen kann oder fügen will. 
nicht ganz unrealistisch wäre) einmal ausklammern, bleibt noch die Frage nach der semantischen Qualität der Codierung.

Die klassische Codierung der Kunst hat auf den Unterschied von schön und hässlich abgestellt, der in sich reflektiert gehandhabt werden muss, das Schöne als abgelehnte Hässlichkeit bzw. als aufgenommene und aufgehobene Hässlichkeit versteht und dritte Möglichkeiten (etwa das Triviale, Banale, Nützliche) als Platzhalter für codefremde Wertungen ausschließt. Schön/hässlich in diesem Verständnis konnte als Leitdifferenz der Kunst angesehen werden, als diejenige Unterscheidung von Unterscheidungen, die dann konkret zum Aufbau des Kunstwerkes dienen. ${ }^{47}$ Da jedoch „,schön“ und „hässlich“ auf Künstler und Kunstkritiker geradezu wie Schreckbegriffe wirken und jedenfalls nicht als angemessene Beschreibung der künstlerischen Tätigkeit empfunden werden, empfiehlt es sich, nicht mit einer Interpretation dieser Begriffe $\mathrm{zu}$ beginnen, sondern das Problem der Codierung auf einem Umwege anzugehen - mit der Frage: wie denn sonst?

Es scheint zwei Möglichkeiten zu geben, den Code schön/hässlich abzuhängen. Die radikalste setzt Code und System gleich. Sie betrachtet die Unterscheidung von zugehörig/nichtzugehörig zur Kunst als einzige noch verbliebene Möglichkeit der Codierung. Damit wird das Prinzip der selbstreferentiellen, autopoietischen Bestimmung radikalisiert: Kunst ist, was sich als Kunst deklariert. Sinn dieser Deklaration ist die Sinnlosigkeit dieser Deklaration. Die in aller Selbstreferenz liegende Letztparadoxie wird zum Ausdruck gebracht. Die Einheit des Systems wird als Objekt dargestellt; das Objekt aber, das dies zum Ausdruck bringen soll, kann nur noch ausgewählte Beliebigkeit sein (denn anderenfalls präsentiert es sich selbst). Wenn dies aber ohne weitere Einschränkungen geschehen soll, erweist sich das Resultat rasch als banal, und es lohnt dann kaum noch, darüber zu kommunizieren, dass gerade dies beabsichtigt war. Eine kurzgeschlossene, nicht über anderes umgeleitete, nicht „entfaltete“ Selbstreferenz gibt für eine Beobachtung nichts mehr her. Sie würde normalerweise den Beobachter veranlassen, seine Beobachtung ihrerseits in Frage zu stellen. Wenn aber genau dies provoziert werden soll und man nur dies noch erfährt, wird man sich fragen, warum man sich gerade damit beschäftigen soll.

Einstweilen ist nicht zu sehen, wie auf der Basis einer solchen Gleichsetzung des Codes mit der Innen/Außen-Differenz des Kunstsystems eine Entwicklung von Kriterien und Programmen eingeleitet werden könnte. Ein Urteil darüber ist sicher verfrüht; aber ebenso sicher ist, dass eine Codierung nur als Aufbauregel für systemeigene Komplexität, nur als genetisches Prinzip jenen Reichtum fort-

47 Siehe auch Luhmann 1981. 
führen bzw. wiedergewinnen kann, auf dem die Ausdifferenzierung der Kunst beruht. ${ }^{48}$ Es besteht daher genug Anlass, sich nach anderen, weniger radikalen Möglichkeiten der Codierung umzusehen.

Nimmt man dabei Tendenzen auf, die sich seit der zweiten Hälfte des 17. Jahrhunderts, wenn nicht früher, durchgesetzt haben, so könnte man an einen Code denken, der Originalität gegen bloßes Kopieren ausspielt. Danach muss jedes Kunstwerk sich in einer Art Überraschungswert, in seiner Distanz zu früheren Kunstwerken bewähren. Auch dieser Code wäre, wie der von schön und hässlich, reflexiv zu handhaben, da die Originalität sich nur an der Vermeidung des Kopierens zeigt, also nahe genug am Kopieren operieren muss und nicht nur irgendetwas anderes anstreben darf. Wie aber wird diese Nähe erzeugt? Und was wird ausgeschlossen dadurch, dass die Kunst sich dem Code Original/Kopie verschreibt?

Über lange Zeit hat es hierfür genügt, sich entweder auf Gattungsmerkmale (ein Sonett hat ein Sonett zu sein) oder auf Stilmerkmale zu verlassen. Wenn diese Führung, aus welchen Gründen immer, aufgegeben wird, muss das Problem, das sie bedient hatte, anders gelöst werden. Wie wird aber, wenn man auf Gattungsfixierungen und auf Stilbindungen verzichtet, erreicht, dass Original und Kopie zusammen einen Code bilden, dass das Original fast eine Kopie hätte sein können, so dass der Code eine Einheit bildet, die deutlich genug gegen andere Möglichkeiten, Artefakte herzustellen, abgrenzt? Genügt es dafür, einen Bildgedanken serienweise auszumalen, bis seine Variationsmöglichkeiten erschöpft sind oder Werksideen so zu individualisieren, dass sie eine begrenzte Menge von familienähnlichen Kunstwerken erzeugen können?

In der neueren Kunst kann man deutlich Tendenzen beobachten, die Unterscheidung von Original und Kopie durch eine strikt temporale Differenz zu ersetzen: Die Kunst darf keinesfalls von gestern sein, und das muss gezeigt werden. Wenn das ein Code werden sollte (woran man berechtigt zweifeln mag), müsste der Präferenzwert der Nichtgestrigkeit, der den Wert der Schönheit ersetzt, in Auseinandersetzung mit der gestrigen Kunst gewonnen werden. Es würde nicht genügen, schlicht anders zu sein; die Andersartigkeit müsste vielmehr, denn sonst wäre es kein Code, in der Auseinandersetzung mit dem Gestrigen gewonnen und an dieser Differenz vorgeführt werden. Das würde aber in der Iteration sehr bald zu der Frage führen, ob das Gestrige, das selbst das Nicht-Vorgestrige, das selbst das Nicht-Vorvorgestrige sein wollte, die Auseinandersetzung noch lohnt. Eine strikt temporale Codierung, und dies wäre denn auch der einzige Fall

48 Diese Einsicht wird nicht allein dadurch schon diskreditiert, dass sie auch für den genetischen Code lebender Systeme gilt. 
eines solchen Versuchs, würde sich selbst durch Iteration ruinieren, besonders wenn noch Tempoerfordernisse und Temposteigerungen hinzukommen.

Vielleicht ist es deshalb nützlich, den Code von Original und Kopie mit Hilfe einer informationstheoretischen Unterscheidung so umzudeuten, dass er deutlicher auf die Eigenkomplexität des einzelnen Kunstwerks bezogen werden kann. In der Informationstheorie unterscheidet man, bezogen auf Systeme, Varietät und Redundanz. Unter Varietät ist die Vielheit der separat bestimmbaren (variierbaren) Elemente eines Kunstwerks zu verstehen, und zwar mehrdimensional nach Maßgabe der benutzten Unterscheidungen unterschieden. Unter Redundanz ist der Prognosewert (bzw. die Minderung des Informationswertes) zu verstehen, die ein Element für andere besitzt - zum Beispiel ein Ton, ein Akkord, eine Tonsequenz für folgende Töne. Redundanz schränkt die Freiheitsgrade für Folgeentscheidungen ein, tendiert also auch dazu, Varietät zu limitieren, weil sie im Maße ihrer Verdichtung immer mehr Möglichkeiten ausschließt. Es gibt mithin ein komplementäres und gegenläufiges Verhältnis von Varietät und Redundanz, wobei Extremwerte (Varietät ohne jede Redundanz und Redundanz ohne jede Varietät) dem Code seinen Reiz nehmen (oder, soziologisch gesehen, Kommunikation aus Anlass von Kunst auf Null reduzieren).

Genauer betrachtet eignet sich diese Unterscheidung von Varietät und Redundanz aber nicht als Code der Kunst. Weder Varietät noch Redundanz können, gleichsam als Nachfolger für „hässlich“, misslungen usw., als Negativwert der Kunstproduktion und des Kunsterlebens dienen. Weder in der einen noch in der anderen Richtung markiert diese Unterscheidung ein Akzeptieren bzw. Verwerfen des Kunstwerks. Vielmehr versucht ein bedeutendes Kunstwerk mit einer Art Leibniz-Formel, so viel Varietät wie möglich mit so viel Redundanz wie nötig zu kombinieren. Das Kunstwerk muss bei hoher Vielfalt noch als Einheit erkennbar sein. Es muss in sich selbst Überraschung und Vertrautheit kombinieren, um der Banalität entgehen und selbst überraschen zu können. ${ }^{49}$ Wenn dafür Anhaltspunkte in Gattungstypik oder Stilmerkmalen, vorgegebener Rhythmik, Sujets

49 Die Anforderungen, die hier erfüllt werden müssen, und die Möglichkeiten der Steigerung von Varietät und Redundanz dürften von Kunstart zu Kunstart sehr verschieden sein. Musik beispielsweise gilt als ein Bereich, der wegen der zeitlichen Erstreckung des Werkes und den Grenzen der Hörfähigkeit mit sehr hohen Redundanzen operieren muss (wie im Übrigen die Sprache auch) (vgl. Meyer 1957). Wenn man dagegen Zeit hat, das Kunstwerk zu betrachten, können „überflüssige“ Redundanzen abgebaut oder vorhandene Redundanzen mit mehr Varietät strapaziert werden. Ambivalent ist der Fall des zu lesenden Kunstwerks, wo man zwar unbegrenzt oft wiederholen und Gedächtnis aufladen könnte, aber dies nicht tut bzw. als nicht zumutbar empfindet. Das Problem der Redundanz firmiert hier als „Spannung“. Und wieder anders liegt der Fall der Lyrik, die mit Kurzzeitgedächtnis rechnen bzw. Nochmallesen zumuten und deshalb in Bezug auf Redundanz größere Risiken eingehen kann. 
usw. nicht mehr ausreichen, muss das Kunstwerk die notwendige Redundanz selbst produzieren, den eigenen Festlegungen gehorchend. Das Unerwartete und Unerwartbare muss durch eine Logik der Selbstbeschränkung als notwendig erscheinen; und dies, wie uns das theoretische Konzept der Unterscheidungen sagt, nicht nur mit dem, was bezeichnet wird, sondern auch mit dem, was damit ausgeschlossen wird, mit den „,anderen Seiten“ der benutzten Unterscheidungen.

Ein solches Steigerungsprinzip ist kein Code, eher eine Gesamtformel für eine Programmatik der Kunst (analog zu Profitmaximierung im Wirtschaftssystem oder Gerechtigkeit im Rechtssystem). Sie ist eine ins systemintern handhabbare übersetzte Beschreibung der Funktion des Systems. Man könnte auch daran denken, das Konzept der regulativen Idee zu übernehmen. Für den durch diese Beschränkung (die noch keine ist) geordneten Bereich differenzieren sich dann Codierung und Programmierung. Der Code organisiert die Doppelmöglichkeit des Akzeptierens und Verwerfens, der Stimmigkeit und der Missstimmigkeit. Als Programm dient die realisierte Redundanz des Systems - sei es vermittelt durch breit akzeptierte Vorstellungen über das, was richtig ist und gefällt, sei es als eine Art Selbstpräjudizierung des einzelnen Kunstwerks.

Je mehr das Kunstwerk sein eigenes Programm wird und werden muss, weil Redundanzvorgaben für Kunst im Allgemeinen versagen, desto unentbehrlicher wird es, Codierung und Programmierung zu unterscheiden. Was richtig ist, mag jeweils dem Einzelwerk überlassen bleiben. Aber dass es überhaupt eine Unterscheidung von Annehmen und Ablehnen geben muss, und zwar eine auf Kunst bezogene Unterscheidung, die nicht mit den politischen, wirtschaftlichen, pädagogischen, wissenschaftlichen und sonstigen Codes verwechselt werden kann, kann man nicht gut bestreiten. Dass die darauf bezogene Semantik von schön/ hässlich nicht mehr als akzeptabel gilt, deutet auf eine Unsicherheit der gesellschaftlichen Bewertung (und eingeschlossen: der Selbstbewertung) von Kunst hin. Wenn man diese Semantik ablehnt, muss man jedoch an genau dieser Funktionsstelle der systemspezifischen Codierung eine andere vorschlagen; man kann es nicht einfach bei der Ablehnung belassen.

\section{Literatur}

Baldus, Claus (1982): Partitives und Distriktives Setzen. Hamburg: Meiner.

Böhringer, Hannes (1985): Die Disziplin der Skepsis. Philosophie am Modell moderner Kunst, in: ders., Begriffsfelder: Von der Philosophie zur Kunst. Berlin: Merve, 73-90.

Bråten, Stein (1983): Asymmetric Discourse and Cognitive Autonomy: Resolving Model Monopoly Through Boundary Shifts, in: Annetta Pedretti/Gerard De Zeeuw (Hrsg.), Problems of Levels and Boundaries. London: Princelet, 7-28. 
Burke, Kenneth (1962): A Grammar of Motives and A Rhetoric of Motives [1945]. Cleveland: World Publishing Company.

Conant, Roger S./Ashby, W. Ross (1970): Every Good Regulator of a System Must be a Model of that System. International Journal of Systems Science 1, 89-97.

Crébillons, Claude (1961): Les égarements du cœur et de l'esprit ou mémoires de M. de Meilcour. Paris: Armand Colin.

d’Alembert, Jean Le Rond (1967): Dialogue entre la poésie et la philosophie, in: ders., CEuvres complètes, Bd. IV [1822], Neudruck. Genf: Slatkine, 373-381.

Ellenberger, Henry T. (1970): The Discovery of the Unconscious: History and Evolution of a Dynamic Psychiatry. New York: Basic Books.

Forster, E.M. (1974): Aspects of the Novel [1927], in: Oliver Stallybras (Hrsg.), The Abinger Edition of E.M. Forster, Bd. 12: Aspects of the Novel and Related Writings. London: E. Arnold.

Günther, Gotthard (1976a): Das metaphysische Problem einer Formalisierung der transzendental-dialektischen Logik, in: ders., Beiträge zur Grundlegung einer operationsfähigen Dialektik, Bd. 1. Hamburg: Meiner, 189-247.

Günther, Gotthard (1976b): Cybernetic Ontology and Transjunctional Operations [1962], in: ders., Beiträge zur Grundlegung einer operationsfähigen Dialektik, Bd. 1. Hamburg: Meiner, 249-328.

Günther, Gotthard (1976c): Formal Logic, Totality and the Super-additive Principle, in: ders., Beiträge zur Grundlegung einer operationsfähigen Dialektik, Bd. 1. Hamburg: Meiner, 329-351.

Günther, Gotthard (1978): Life as Poly-Contexturality [1973] in: ders., Beiträge zur Grundlegung einer operationsfähigen Dialektik, Bd. 2. Hamburg: Meiner, 283-306.

Heidegger, Martin (1950): Der Ursprung des Kunstwerkes, in: ders., Holzwege. Frankfurt a.M.: Klostermann, 7-68.

Husserl, Edmund (1948): Erfahrung und Urteil. Untersuchungen zur Genealogie der Logik. Hamburg: Claasen \& Goverts.

Kahneman, Daniel/Tversky, Amos (1984): Choices, Values, and Frames. American Psychologist 39, 341-350.

Le Mercier de La Rivière, Paul-Pierre (1767): De l'ordre naturel et essentiel des sociétés politiques. Paris/London: Nourse/Desaint.

Luhmann, Niklas (1981): Ist Kunst codierbar?, in: ders., Soziologische Aufklärung 3: Soziales System, Gesellschaft, Organisation. Opladen: Westdeutscher Verlag, 245-266.

Luhmann, Niklas (1986a): Die Lebenswelt - nach Rücksprache mit Phänomenologen. Archiv für Rechts- und Sozialphilosophie 72, 176-194.

Luhmann, Niklas (1986b): Ökologische Kommunikation. Kann die moderne Gesellschaft sich auf ökologische Gefährdungen einstellen? Opladen: Westdeutscher Verlag.

Luhmann, Niklas (1986c): „Distinctions directrices“: Über Codierung von Semantiken und Systemen, in: Friedhelm Neidhardt/M. Rainer Lepsius/Johannes Weiß (Hrsg.), Kultur und Gesellschaft. Opladen: Westdeutscher Verlag, 145-161 [Wiederabdruck in: ders. (1987), Soziologische Aufklärung 4: Beiträge zur funktionalen Differenzierung der Gesellschaft. Opladen: Westdeutscher Verlag, 13-31].

Mackay, Donald M. (1964): Communication and Meaning: A Functional Approach, in: F.S.C. Northrop/Helen H. Tivingston (Hrsg.), Cross-Cultural Understanding: Epistemology in Anthropology. New York: Harper \& Row, 162-179.

Maturana, Humberto R. (1982): Biologie der Kognition, in: ders., Erkennen: Die Organisation und Verkörperung von Wirklichkeit. Ausgewählte Arbeiten zur biologischen Epistemologie. Braunschweig: Vieweg, 32-80. 
Merleau-Ponty, Maurice (1964): Le visible et l'invisible. Suivi de notes de travail. Paris:

Gallimard.

Meyer, Eva (1983): Zählen und Erzählen: Für eine Semiotik des Weiblichen. Wien/Berlin: Medusa.

Meyer, Leonard B. (1957): Meaning in Music and Information Theory. Journal of Aesthetics 15, 412-424 [neu gedruckt in: ders. (1967), Music, The Arts, and Ideas. Chicago: University of Chicago Press, 5-21].

Parsons, Talcott (1978): A Paradigm of the Human Condition, in: ders., Action Theory and the Human Condition. New York: Free Press, 352-433.

Pask, Gordon (1981): Organizational Closure of Potentially Conscious Systems, in: Milan Zeleny (Hrsg.), Autopoiesis: A Theory of Living Organization. New York: North-Holland, 265-308.

Rotter, Frank (1985): Musik als Kommunikationsmedium: Soziologische Medientheorien und Musiksoziologie. Berlin: Duncker \& Humblot.

Serres, Michel (1981): Der Parasit. Frankfurt a.M.: Suhrkamp.

Simon, Herbert A. (1962): The Architecture of Complexity. Proceedings of the American Philosophical Society 106, 467-482.

Simon, Herbert A. (1967): The Logic of Heuristic Decision Making, in: Nicholas Rescher (Hrsg.), The Logic of Decision and Action. Pittsburgh: University of Pittsburgh Press, 1-20.

Spencer Brown, George (1979): Laws of Form [1969], Neudruck der 2. Aufl. (1971). New York: Dutton.

Stockton, Frank R. (1969): The Lady, or the Tiger? [1884], in: ders., The Lady, or the Tiger? And other Stories, Nachdruck. New York: Garrett Press, 1-10.

Valéry, Paul (1931): L'âme et la danse [1921], in: ders., L’âme et la danse - Eupalinos, ou l'architecte - Paradoxe sur l'architecte. Paris: Sagittaire, 11-63.

van den Berg, J.H. (1974): Divided Existence and Complex Society: A Historical Approach. Pittsburgh: Duquesne University Press.

Van Parijs, Philippe (1977): Triadic Distributions and Contrepied Strategies: A Contribution to a Pure Theory of Expressive Behaviour. Journal for the Theory of Social Behaviour 7, 129-160.

Varela, Francisco J. (1975): A Calculus for Self-Reference. International Journal of General Systems 2, 5-24.

von Foerster, Heinz (1979): Cybernetics of Cybernetics, in: Klaus Krippendorff (Hrsg.), Communication and Control in Society. New York: Gordon and Breach, 5-8.

von Foerster, Heinz (1981): Observing Systems. Seaside, Cal.: Intersystems Publications.

Waldenfels, Bernhard (1979): Die Abgründigkeit des Sinnes: Kritik an Husserls Idee der Grundlegung, in: Elisabeth Ströker (Hrsg.), Lebenswelt und Wissenschaft in der Philosophie Edmund Husserls. Frankfurt a.M.: Klostermann, 124-142 [neu gedruckt in: ders. (1985), In den Netzen der Lebenswelt. Frankfurt a.M: Suhrkamp, 15-33].

Weick, Karl E. (1985): Der Prozeß des Organisierens. Frankfurt a.M.: Suhrkamp. 